\title{
On the Price of Risk Under a Regime Switching CGMY Process
}

\author{
Pious Asiimwe ${ }^{1}$. Charles Wilson Mahera ${ }^{2}$. \\ Olivier Menoukeu-Pamen ${ }^{3}$
}

(C) The Author(s) 2016. This article is published with open access at Springerlink.com

\begin{abstract}
In this paper, we study option pricing under a regime-switching exponential Lévy model. Assuming that the coefficients are time-dependent and modulated by a finite state Markov chain, we generalise the work in Momeya and Morales (Method Comput Appl Probab, 2014, doi:10.1007/s11009-014-9399-2), and Siu and Yang (Acta Mathe Appl Sin 2:369-388, 2009), that is, we use a pricing method based on the Esscher transform conditional on the information available on the Markov chain. We also carry out numerical analysis, to show the impact of the risk induced by the underlying Markov chain on the price of the option.
\end{abstract}

\footnotetext{
This work is based on Pious Asiimwe's MSc Dissertation from University of Dar es Salaam. He acknowledges the financial support by NORAD through its NOMA program. The research of Olivier Menoukeu-Pamen was supported by the LMS (London Mathematical Society) grant number 51305 . He also thanks the Department of Mathematics, University of Dar es Salaam for their hospitality and for providing nice work environment.

$凶$ Olivier Menoukeu-Pamen

Menoukeu@liv.ac.uk

Pious Asiimwe

asiimwepious88@hotmail.com

Charles Wilson Mahera

cwmahera@aims.ac.tz

1 Department of Mathematics, University of Dar es Salaam, P.O. Box 35062, Dar es Salaam, Tanzania

2 African Institute of Mathematical Sciences, Bagamoyo, Tanzania

3 Institute for Financial and Actuarial Mathematics, Department of Mathematical Sciences, University of Liverpool, Liverpool L15 8HB, UK
} 
Keywords Option pricing · Regime switching risk · Exponential Lévy model • Regime switching Esscher transform

Mathematics Subject Classification 91G60 • 91G20 • 60G44 · 60G51

\section{Introduction}

Empirical studies have suggested the need for modern financial modelling to move from the standard log-normal dynamics of the Black-Scholes model framework. This is primarily because in their works, the authors in Black and Scholes (1973); Merton (1973) assume that the price dynamic of the underlying risky asset are governed by geometric Brownian motion, an assumption which many researchers have challenged. There is evidence that the risky assets experience stochastic volatility overtime and therefore the assumption of constant volatility creates biases when an option is priced using the Black-Scholes model. Several models have been developed to provide more realistic ways to model empirical behaviour of option prices. Among them, we can list: the jump-diffusion models, the stochastic volatility and the regime switching models. In the latter case, economic cycles are described by a discrete, finite state Markov chain; See for example Goldfeld and Quandt (1973); Hamilton (1989) for more details. The states of the underlying Markov chain represent the different states of the economy and such model enable to incorporate the impact of changes in macro-economic conditions on the behaviour of the dynamics of the assets' prices.

The possibility of switching across induces an important source of risk that investors might want to hedge against. As pointed out in Elliott et al. (2011), in a regime switching Black-Scholes model, there exist at least two sources of risk that the investor needs to consider: the diffusion risk which can be considered as the market or financial risk and regime switching risk which can be thought as economic risk. In addition, when the underlying is driven by a Lévy process, one needs to consider the risk due to multiple jumps coming from Poisson random measures. There has been many works on option pricing under regime switching model, most of them assuming that the risk due to switching of regimes is zero. In Siu and Yang (2009); Elliott and Siu (2011); Momeya and Morales (2014), the importance of pricing the regime risk is shown, in the sense that, the authors show the impact of the change in the regime on the option prices, hence addressing the problem of pricing the risk associated to the regime. The work regime switching Black-Scholes model is discussed in Siu and Yang (2009); Elliott and Siu (2011) whereas Momeya and Morales (2014) is an extension to the regime-switching Variance-Gamma model. See also the work Naik (1993) where the author studies the price of the regime risk induced by the jumps in volatility.

One of the main characteristics of the regime-switching model is that they generate incomplete market and hence a family of Equivalent Martingales Measure (EMM). The first task is to determine an equivalent martingale measure which will enable to price the different risks efficiently. One may think of the martingale measure that minimises the "distance" between the set of equivalent martingale measures and the real world probability measure. One of such distances is given by the relative entropy and the associated minimiser is the minimal entropy martingale measure (MEMM). In 
this work, we will use the regime switching Esscher transform which was already used in Siu and Yang (2009) (see also Momeya and Morales 2014). The Esscher transform is taken conditional on the information available on the Markov chain. The result by Momeya and Ben-Salah (2012) can be used to justify the choice of our pricing result by the minimal entropy martingale measure. It is also worth mentioning that the work Gerber and Shiu (1994) introduces Esscher transform in actuarial science as the pricing measure for option valuation and justify this choice by maximizing the expected utility of power type of an investor. For other works on minimal entropy martingale measure, the reader may consult Arai (2001); Fujiwara (2006); Fujisak and Zhang (2009); Miyahara (1999).

In this paper, we extend the works Momeya and Morales (2014); Siu and Yang (2009), that is, we assume that the dynamic of the underlying risky asset is governed by a regime switching Carr, Geman, Madan and Yor (CGMY) process. We first study the option price under a general regime switching exponential Lévy model. In this model, the parameters of the assets are assumed to be deterministic, time dependent and are modulated by an observable continuous time, finite state Markov chain. For example, one may interpret the time dependent interest rate as corresponding to the relative frequent announcements or industry involving reasonably small shifts in the interest rates (see for example Hyland et al. 1999). One may also interpret the observable states of the chain as different stages of the business cycle, for instance if the states of the Markov chain are two, they could be interpreted as expansion and recession periods. As in Momeya and Morales (2014); Siu and Yang (2009), we introduce a pricing model to price the diffusion risk (for the time dependent regime switching Black-Scholes model), the risk due to jumps and the regime-switching risk. To achieve this, we first adopt the regime switching Esscher transform in order to determine a set of equivalent martingale measures satisfying the martingale condition. The selection of the Esscher transform martingale measure is done by minimizing the maximum entropy between an equivalent martingale measure and the real world probability measure over the different states of the economy (compare with Siu and Yang 2009; Momeya and Morales 2014).

We conduct numerical experiments to show the impact of the risk induced by the underlying Markov chain on the price of the option. This implies that in pricing options, a probable error can be made when we chose to ignore the risk associated with the switching of regimes. Our results extend those in Siu and Yang (2009); Momeya and Morales (2014) to incorporate the time dependency of the parameters and to the CGMY model. Another interesting observation in our model is the following: During the lifetime of the option, its price is higher when the regime risk is priced than when it is not, which is higher than the option price when there is no regime.

The remaining of the paper is organized as follows: In Sect. 2, we describe the model and study the different pricing kernels and their associated martingale condition. These conditions are explicitly given is the case of regime switching Black-Scholes model, Variance-Gamma and CGMY model. Section 3 is devoted to numerical experiments to illustrate the effect of pricing the regime switching risk and we find a significant difference between pricing the risk and not. 


\section{The Model}

In this section, we present a general regime switching exponential Lévy model. The model is similar to that of Momeya and Morales (2014). Let $(\Omega, \mathcal{F}, \mathbb{P})$ be a complete probability space, where $\mathbb{P}$ is the reference measure.

The evolution of the states of the economy is modelled by an irreducible homogeneous continuous time Markov chain $X:=\{X(t) ; t \in[0, T]\}$ with a finite state space $\mathcal{X}=\left\{\mathbf{e}_{1}, \mathbf{e}_{2}, \ldots, \mathbf{e}_{N}\right\} \in \mathbb{R}^{N}$, where $N \in \mathbb{N}$, and the $j$ th component of $e_{n}$ is the Kronecker delta $\delta_{n j}$ for each $n, j=1, \ldots, D$. Denote by $\mathbf{A}:=\left[a_{i j}\right]_{i, j=1,2, \ldots, N}$ the intensity matrix of the Markov chain under $\mathbb{P}$. Then for each $i, j=1,2, \ldots, N$ with $i \neq j, a_{i j}$ is the transition intensity of the chain $X$ jumping from state $\mathbf{e}_{j}$ to state $\mathbf{e}_{i}$ at time $t \in[0, T]$. Hence, for $i \neq j, a_{i j} \geq 0$ and $\sum_{j=1}^{N} a_{i j}=0$ i.e., $\lambda_{i i} \leq 0$. With the canonical representation of the state space of the Markov chain, the following semimartingale decomposition for the Markov chain $X$ was given in Elliott et al. (1994):

$$
X(t)=X(0)+\int_{0}^{t} A(s) X(s) \mathrm{d} s+M(t), \quad t \in[0, T] .
$$

where $\{M(t) ; t \in[0, T]\}$ is an $\mathbb{R}^{N}$-valued martingale under the measure $\mathbb{P}$ with respect to the filtration generated by $X$.

We consider a financial market with two primary securities, namely, a riskless asset $B$ and a risky stock $S$, which are traded continuously over the time horizon $[0, T]$. We model the evolution of the instantaneous interest rate $r=\{r(t) ; t \in[0, T]\}$ of the money market account $B$ at time $t$ as follows.

$$
r(t)=r(t, X(t))=\langle\mathbf{r}, X(t)\rangle=\sum_{i=1}^{N} r_{i}(t)\left\langle\mathbf{e}_{i}, X(t)\right\rangle,
$$

where $\mathbf{r}:=\left(r_{1}(t), r_{2}(t), \ldots, r_{N}(t)\right)^{\prime} \in \mathbb{R}^{N}$ for each $i=1,2, \ldots, N$ and $\langle\cdot, \cdot\rangle$ denotes the inner product in $\mathbb{R}^{N}$. The $\mathrm{i}$-th component $r_{i}(t)$ of the vector $\mathbf{r}$ is a deterministic function, representing the value of the interest rate when the Markov chain is in state $\mathbf{e}_{i}$ that is when $X(t)=\mathbf{e}_{i}$. The dynamics of $\{B(t) ; t \in[0, T]\}$ of the money market account $B$ are given by

$$
\mathrm{d} B(t)=r(t) B(t) \mathrm{d} t, \quad B(0)=1 .
$$

Denote by $\{\mu(t) ; t \in[0, T]\}$ and $\{\sigma(t) ; t \in[0, T]\}$ the appreciation rate and the volatility of the stock $S$ at the time $t$ respectively. Using similar convention, we set

$$
\begin{aligned}
& \mu(t)=\mu(t, X(t)):=\langle\boldsymbol{\mu}, X(t)\rangle=\sum_{i=1}^{N} \mu_{i}(t)\left\langle\mathbf{e}_{i}, X(t)\right\rangle, \\
& \sigma(t)=\sigma(t, X(t)):=\langle\sigma, X(t)\rangle=\sum_{i=1}^{N} \sigma_{i}(t)\left\langle\mathbf{e}_{i}, X(t)\right\rangle,
\end{aligned}
$$


where $\boldsymbol{\mu}=\left(\mu_{1}(t), \mu_{2}(t), \ldots, \mu_{N}(t)\right)^{\prime} \in \mathbb{R}^{N}$ and $\boldsymbol{\sigma}=\left(\sigma_{1}(t), \sigma_{2}(t), \ldots, \sigma_{N}(t)\right)^{\prime} \in$ $\mathbb{R}_{+}^{N}$.

$\mu_{i}(t)$ and $\sigma_{i}(t), i=1,2 \ldots, N$ are deterministic functions representing respectively the appreciation rate and volatility of $S$ when the Markov chain is in state $\mathbf{e}_{i}$. The price dynamics of the stock $S$ is given by the following stochastic differential equation:

$$
\mathrm{d} S(t)=S\left(t^{-}\right)\left(\mu(t) \mathrm{d} t+\sigma(t) \mathrm{d} W(t)+\int_{\mathbb{R}_{0}}\left(e^{z}-1\right) \tilde{N}^{X}(\mathrm{~d} t ; \mathrm{d} z)\right), \quad S(0)>0,
$$

where $\mathbb{R}_{0}=\mathbb{R} \backslash\{0\}, W=\{W(t) ; t \in[0, T]\}$ is a Brownian motion and $\widetilde{N}^{X}(\mathrm{~d} t, \mathrm{~d} z):=N(\mathrm{~d} z, \mathrm{~d} t)-\rho^{X}(\mathrm{~d} z) \mathrm{d} t$ is an independent compensated Markov regimeswitching Poisson random measure with $\rho^{X}(\mathrm{~d} z) \mathrm{d} t$, the compensator (or dual predictable projection) of $N$, defined by:

$$
\rho^{X}(\mathrm{~d} z) \mathrm{d} t:=\sum_{j=1}^{D}\left\langle X(t-), e_{j}\right\rangle \rho_{j}(\mathrm{~d} z) \mathrm{d} t
$$

For each $j \in\{1,2, \ldots, D\}, \rho_{j}(\mathrm{~d} z)$ is the conditional density of the jump size when the Markov chain $X$ is in state $e_{j}$ and satisfies $\int_{\mathbb{R}_{0}} \min \left(1, z^{2}\right) \rho_{j}(\mathrm{~d} z)<\infty$ and $\int_{|z| \geq 1}\left(e^{z}-1\right)^{2} \rho_{i}(z) \mathrm{d} z<\infty$.

The dynamic of the stock $S$ can also be written as

$$
S(t)=S(0) e^{Y(t)}
$$

where $Y(t)$ is given by:

$$
\begin{aligned}
Y(t) & =Y(0)+\int_{0}^{t}\left(\mu(s)-\frac{1}{2} \sigma^{2}(s)-\int_{\mathbb{R} \backslash\{0\}}\left(e^{z}-1-z\right) \rho^{X}(\mathrm{~d} z)\right) \mathrm{d} s \\
& +\int_{0}^{t} \sigma(s) \mathrm{d} W(s)+\int_{0}^{t} \int_{\mathbb{R} \backslash\{0\}} z \tilde{N}(\mathrm{~d} s, \mathrm{~d} z) .
\end{aligned}
$$

The model defined by (2.1)-(2.6) is referred to as a general regime switching exponential Lévy model. Such model leads to incomplete markets i.e., there exists more than one equivalent martingale measures (EMM) describing the risk-neutral price dynamic and compatible with the no arbitrage requirement. In order to price contingent claim, we shall determine EMM using regime switching Esscher transform introduced in Elliott et al. (2005); Siu and Yang (2009). In fact, the classical definition of Esscher transform based on the moment generating function of a random variable is replaced by a conditional Esscher transform where the moment generating function is conditional to a subset of information available on the Markov chain. This leads to two different pricing kernels based on the conditional Esscher transform. 


\subsection{Pricing Kernel I}

In this section, we construct a risk neutral measure assuming that the whole path of the underlying Markov chain is known. This Esscher change of measure produces a pricing kernel that does not take into account the risk associated with the Markov chain.

We shall first specify the information structure of our model. Let $F^{X}:=\left\{\mathcal{F}_{t}^{X} ; t \in\right.$ $[0, T]\}$ and $F^{S}:=\left\{\mathcal{F}_{t}^{S} ; t \in[0, T]\right\}$ denote the $\mathbb{P}$-augmentation of natural filtrations generated by $\{X(t) ; t \in[0, T]\}$ and $\{S(t) ; t \in[0, T]\}$ respectively. That is, for each $t \in[0, T], \mathcal{F}_{t}^{X}$ and $\mathcal{F}_{t}^{S}$ are, respectively, the $\sigma$-fields generated by the histories of the chain $X$ and the stock price $S$ up to and including time $t$. We define for $t \in[0, T], \mathcal{G}_{t}$ to be the $\sigma$-algebra $\mathcal{F}_{T}^{X} \vee \mathcal{F}_{t}^{S}$. This represents the information set generated by both histories of $X$ and $S$ up to and including the time $t$. We write $\mathbf{G}:=\left\{\mathcal{G}_{t} ; t \in[0, T]\right\}$. We set

$\Theta:=\left\{\theta(t) ; t \in[0, T] \mid \theta(t):=\sum_{i=1}^{N} \theta_{i}(t)\left\langle X\left(t^{-}\right), \mathbf{e}_{i}\right\rangle\right.$, with $\left(\theta_{1}(t), \ldots, \theta_{N}(t)\right) \in \mathbb{R}^{N}$ such that $\theta_{i}, i=1, \ldots, N$ are deterministic and $\left.E^{\mathbb{P}}\left[e^{-\int_{0}^{t} \theta(s) \mathrm{d} Y(s)} \mid \mathcal{F}_{T}^{X}\right]<\infty\right\}$.

For $\theta:=\{\theta(t) ; t \in[0, T]\} \in \Theta$, define the generalized Laplace transform of a G-adapted process $Y$ by

$$
M_{Y}(\theta):=E^{\mathbb{P}}\left[e^{-\int_{0}^{t} \theta(s) \mathrm{d} Y(s)} \mid \mathcal{F}_{T}^{X}\right]
$$

We define the kernel of a generalized Esscher transform with respect to the parameter $\theta$. Let $\Lambda^{\theta}:=\left\{\Lambda^{\theta}(t) ; t \in[0, T]\right\}$ denote a $\mathbf{G}$-adapted stochastic process defined as

$$
\Lambda^{\theta}(t)=\frac{\exp \left(-\int_{0}^{t} \theta(s) \mathrm{d} Y(s)\right)}{M_{Y}(\theta)}, \quad t \in[0, T], \quad \theta \in \Theta .
$$

Then, the regime switching Esscher transform $\mathbb{Q} \sim \mathbb{P}$ on $\mathbf{G}$ with respect to a family of parameters $\{\theta(s) ; s \in[0, t]\}$ is given by:

$$
\Lambda^{\theta}(t)=\left.\frac{\mathrm{d} \mathbb{Q}}{\mathrm{d} \mathbb{P}}\right|_{\mathcal{G}_{t}}=\frac{\exp \left(-\int_{0}^{t} \theta(s) \mathrm{d} Y(s)\right)}{E_{\mathbb{P}}\left[\exp \left(-\int_{0}^{t} \theta(s) \mathrm{d} Y(s)\right) \mid \mathcal{F}_{T}^{X}\right]}, \quad t \in[0, T], \quad \theta \in \Theta .
$$


Hence, as shown in Elliott et al. (2005), one has

$$
\begin{aligned}
\Lambda^{\theta}(t)= & \exp \left(-\int_{0}^{t} \theta(s) \sigma(s) \mathrm{d} W(s)-\frac{1}{2} \int_{0}^{t}(\theta(s))^{2}(\sigma(s))^{2} \mathrm{~d} s\right. \\
& \left.-\int_{0}^{t} \int_{\mathbb{R}_{0}} \theta\left(s^{-}\right) z \tilde{N}^{X}(\mathrm{~d} s, \mathrm{~d} z)-\int_{0}^{t} \int_{\mathbb{R}_{0}}\left(e^{-z \theta(s)}-1+\theta(s) z\right) \rho^{X}(\mathrm{~d} z) \mathrm{d} s\right) .
\end{aligned}
$$

For each $\theta \in \Theta, \Lambda^{\theta}$ is a density process (see Momeya and Morales 2014; Siu and Yang 2009), therefore a new equivalent probability measure can be defined by setting

$$
\left.\frac{\mathrm{d} \mathbb{Q}^{\theta}}{\mathrm{d} \mathbb{P}}\right|_{\mathcal{G}_{t}}=\Lambda^{\theta}(t), \quad t \in[0, T] .
$$

The pricing kernel associated to such measure shall then be defined by choosing $\theta$ adequately (see Sect. 2.3).

\subsection{Pricing Kernel II}

In this section, we construct a change of measure assuming that the initial state of the underlying Markov chain is known. This assumption seems more realistic since an investor can only observe the current and past information about the macro-economic condition and then anticipate future evolution of the macro-economic conditions. The expectation in the denominator of the regime switching Esscher transform is unconditional implying that the risk due to the switching regimes is priced.

We introduce a new filtration, namely $\mathbb{G}:=\left\{\overline{\mathcal{G}}_{t}=\mathcal{F}_{t}^{X} \vee \mathcal{F}_{t}^{S} ; t \in[0, T]\right\}$ which denotes the right continuous, $\mathbb{P}$-complete filtration generated by the bivariate process $(X, S)$. Set

$$
\begin{aligned}
\Theta^{*}:=\left\{\theta^{*}(t) ; t \in[0, T] \mid \theta^{*}(t)\right. & :=\sum_{i=1}^{N} \theta_{i}^{*}(t)\left\langle X\left(t^{-}\right), \mathbf{e}_{i}\right\rangle, \text { with } \\
& \left.\left(\theta_{1}^{*}(t), \ldots, \theta_{N}^{*}(t)\right) \in \mathbb{R}^{N}, \text { such that } E^{\mathbb{P}}\left[e^{-\int_{0}^{T} \theta^{*}(s) \mathrm{d} Y(s)} \mid X(0)\right]<\infty\right\}
\end{aligned}
$$

and define the generalized Laplace transform of a $\mathbb{G}$-adapted process $Y$ as

$$
M_{Y}\left(\theta^{*}\right):=E^{\mathbb{P}}\left[e^{-\int_{0}^{T} \theta(s) \mathrm{d} Y(s)} \mid X(0)\right]
$$

As in Momeya and Morales (2014); Siu and Yang (2009), define the new kernel $\Lambda^{\theta^{*}}=\left\{\Lambda^{\theta^{*}}(t) ; t \in[0, T]\right\}$ as follows 


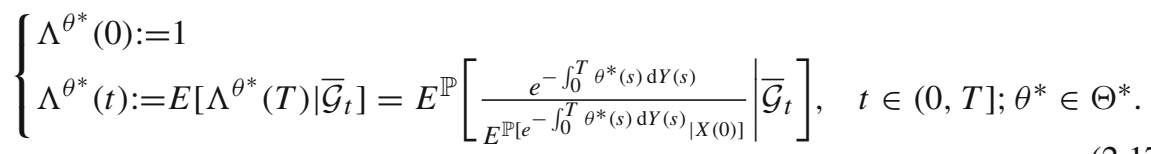

Then $\left\{\Lambda^{\theta^{*}}(t) ; t \in[0, T]\right\}$ is a positive $(\mathbb{G}, \mathbb{P})$-martingale satisfying

$$
E^{\mathbb{P}}\left[\Lambda^{\theta^{*}}\right]=1, \quad \forall t \in[0, T] .
$$

As for the first kernel, one can define a family of equivalent measures $\mathbb{Q}_{\theta^{*}}$ through

$$
\frac{\mathrm{d} \mathbb{Q}_{\theta^{*}}}{\mathrm{~d} \mathbb{P}} \mid \overline{\mathcal{G}}_{t}=\Lambda^{\theta^{*}}(t), \quad t \in[0, T] .
$$

and derive a pricing kernel by adequately choosing $\theta^{*}$ (see Sect. 2.3.)

The pricing kernel (2.14) and (2.18) and The knowledge of the whole path of the Markov chain implies that there is no need for additional premium whereas the knowledge of only the initial state of the Markov chain forces the need of additional premium that will take into account the risk associated to the changes in the regime.

\subsection{Martingale Condition}

Denote by $\left\{S^{*}(t):=\frac{S(t)}{B(t)} ; t \in[0, T]\right\}$ the discounted price process. Therefore, by the fundamental theorem of asset pricing (see Harrison and Pliska 1981, 1983), the noarbitrage price of any contingent claim written on $S$ in this market is given by

$$
E^{\mathbb{Q}}\left[S^{*}(t) \mid \mathcal{G}_{0}\right]=S^{*}(0)
$$

with $\mathbb{Q} \in\left\{\mathbb{Q}^{\theta}, \mathbb{Q}_{\theta^{*}}\right\}$. Eq. (2.19) implicitly gives the condition on the process $\theta$ and $\theta^{*}$ that determine an EMM within the families $\left\{\mathbb{Q}^{\theta}: \theta \in \Theta\right\}$ and $\left\{\mathbb{Q}_{\theta^{*}}: \theta^{*} \in \Theta^{*}\right\}$.

The following theorem gives necessary and sufficient conditions for $\mathbb{Q}^{\theta}$ to be an EMM.

Theorem 2.1 Consider the Lévy regime-switching market defined in (2.3) and (2.6). An equivalent probability measure $\mathbb{Q}^{\theta}$ defined through (2.14) is an equivalent martingale measure on $\left(\Omega, \mathcal{G}_{T}\right)$,i.e., it satisfies the condition (2.19), if and only if $\theta$ satisfies the following equation

$$
\begin{aligned}
& \mu_{i}(t)-r_{i}(t)-\theta_{i}(t) \sigma_{i}^{2}(t)+\int_{\mathbb{R}}\left(e^{z}-1\right)\left(e^{-z \theta_{i}(t)}-1\right) \rho_{i}(z) \mathrm{d} z=0, \\
& t \text {-a.e., } \forall t \in[0, T]
\end{aligned}
$$

for $i=1, \ldots, N$.

Proof It easily follows using the martingale condition under the enlarged filtration $\mathbf{G}=\left\{\mathcal{G}_{t} ; 0 \leq t \leq T\right\}$ and Bayes rules. 
Next, we shall discuss the necessary and sufficient condition for $\mathbb{Q}_{\theta^{*}}$ to be an equivalent martingale on $\left(\Omega, \mathcal{G}_{T}\right)$. We begin by presenting, without proof, a lemma which gives an explicit form of the moment generating function of the Markov chain in terms of the occupation times.

Lemma 2.2 Consider an irreducible homogeneous continuous-time Markov chain $X:=\{X(t) ; t \in[0, T]\}$ on $\left(\Omega, \mathcal{G}_{T}, \mathbb{G}, \mathbb{P}\right)$ with a finite state space $\mathcal{X}$ of size $N \in \mathbb{N}$ and with an intensity matrix $\boldsymbol{A}:=\left\{a_{i j}: 1 \leq i, j \leq N\right\}$. Let

$$
\underline{\mathbf{J}}(u, v):=\left(J_{1}(u, v), J_{2}(u, v), \ldots, J_{N}(u, v)\right)
$$

denote the vector of the occupation times of $X$ during a period of time $[u, v] \subset[0, T]$. We have

$$
J_{k}(u, v)=\int_{u}^{v}\left\langle X(s), \mathbf{e}_{k}\right\rangle \mathrm{d} s .
$$

The conditional moment generating function of $\underline{\mathbf{J}}(u, v)$ is given by

$$
E^{\mathbb{P}}\left[e^{\sum_{k=1}^{N} \int_{u}^{t} \zeta_{k}(v) \mathrm{d} J_{k}(u, v)} \mid \mathcal{G}_{u}\right]=\left\langle e^{\int_{u}^{t}\left(A+\operatorname{Diag}\left(\underline{\zeta}_{k}(r)\right)\right) \mathrm{d} r} X(u), \underline{\mathbf{1}}\right\rangle, \quad \underline{\zeta} \in \mathbb{R}^{N},
$$

where $\underline{\mathbf{1}}=(1,1, \ldots, 1)^{\prime} \in \mathbb{R}^{N},\langle\cdot, \cdot\rangle$ is the scalar product in $\mathbb{R}^{N}$ and Diag $(\underline{\zeta})$ is an $N \times N$ diagonal matrix of the form

$$
\operatorname{Diag}(\underline{\zeta})=\left(\begin{array}{ccccc}
\zeta_{1} & 0 & \ldots & 0 & 0 \\
0 & \zeta_{2} & 0 & \ldots & 0 \\
\vdots & \ddots & \ddots & \ddots & \vdots \\
\vdots & \ddots & \ddots & \zeta_{N-1} & 0 \\
0 & \ldots & 0 & 0 & \zeta_{N}
\end{array}\right)
$$

Proof Follows in the same way as in the proof of Elliott and Osakwe (2006) Proposition 2 .

We can now state the necessary and sufficient condition for $\mathbb{Q}_{\theta^{*}}$ to be an equivalent martingale measure on $\left(\Omega, \mathcal{G}_{T}\right)$. This result is adapted from Siu and Yang Siu and Yang (2009).

Theorem 2.3 Consider the Lévy regime-switching market defined in (2.3) and (2.6). An equivalent measure $\mathbb{Q}_{\theta^{*}}$ defined through (2.18) is an equivalent martingale measure on $\left(\Omega, \mathcal{G}_{T}\right)$, i.e., condition (2.19) holds if and only if $\theta^{*}$ satisfies the following equation

$$
\left\langle e^{\int_{0}^{t}\left(\boldsymbol{A}+\operatorname{Diag}\left(\underline{\tilde{\xi}}\left(\theta^{*}(r)\right)\right) \mathrm{d} r\right.} X(0), \underline{\mathbf{1}}\right\rangle-\left\langle e^{\int_{0}^{t}\left(\boldsymbol{A}+\operatorname{Diag}\left(\underline{\xi}\left(\theta^{*}(r)\right)\right) \mathrm{d} r\right.} X(0), \underline{\mathbf{1}}\right\rangle=0,
$$

where

$$
\begin{aligned}
& \underline{\xi}\left(\theta^{*}\right)=\left(\xi_{1}\left(\theta_{1}^{*}(t)\right), \xi_{2}\left(\theta_{2}^{*}(t)\right), \ldots, \xi_{N}\left(\theta_{N}^{*}(t)\right)\right), \\
& \underline{\tilde{\xi}}\left(\theta^{*}\right)=\left(\tilde{\xi}_{1}\left(\theta_{1}^{*}(t)\right), \tilde{\xi}_{2}\left(\theta_{2}^{*}(t)\right), \ldots, \tilde{\xi}_{N}\left(\theta_{N}^{*} t\right)\right),
\end{aligned}
$$


with

$$
\begin{aligned}
\xi_{i}\left(\theta_{i}^{*}(t)\right)= & -\theta_{i}^{*}(t)\left(\mu_{i}(t)-\frac{1}{2} \sigma_{i}^{2}(t)\right)+\frac{1}{2}\left(\theta_{i}^{*}(t)\right)^{2} \sigma_{i}^{2}(t) \\
& +\int_{\mathbb{R}}\left(e^{-z \theta_{i}^{*}(t)}-1+\theta_{i}^{*}(t)\left(e^{z}-1\right)\right) \rho_{i}(z) \mathrm{d} z, t \text {-a.e. }, \\
\tilde{\xi}_{i}\left(\theta_{i}^{*}(t)\right)= & -r_{i}(t)-\left(\theta_{i}^{*}(t)-1\right)\left(\mu_{i}(t)-\frac{1}{2} \sigma_{i}^{2}(t)\right)+\frac{1}{2}\left(\theta_{i}^{*}(t)-1\right)^{2} \sigma_{i}^{2}(t) \\
& +\int_{\mathbb{R}}\left(e^{-z\left(\theta_{i}^{*}(t)-1\right)}-1+\left(\theta_{i}^{*}(t)-1\right)\left(e^{z}-1\right)\right) \rho_{i}(z) \mathrm{d} z, t \text {-a.e. }
\end{aligned}
$$

for $i=1,2, \ldots, N$.

In order to prove this theorem, we will need the following lemma, which is a extension of (Momeya and Morales 2014 Lemma 4.2) and (Siu and Yang 2009 Lemma 3.1).

Lemma 2.4 Under Assumptions of Theorem 2.3, for all $u, v \in[0, T]$ such that $u \leq v$, we have that

$$
E^{\mathbb{Q}_{\theta^{*}}}\left[S^{*}(v) \mid \mathcal{G}_{u}\right]=\frac{\left\langle e^{\int_{u}^{v}\left(\boldsymbol{A}+\operatorname{Diag}\left(\tilde{\xi}\left(\theta^{*}(r)\right)\right) \mathrm{d} r\right.} X(u), \underline{\mathbf{1}}\right\rangle}{\left\langle e^{\int_{u}^{v}\left(\boldsymbol{A}+\operatorname{Diag}\left(\underline{\xi}\left(\theta^{*}(r)\right)\right)\right) \mathrm{d} r} X(u), \underline{\mathbf{1}}\right\rangle} S^{*}(u),
$$

where $\underline{\xi}\left(\theta^{*}(r)\right)$ and $\underline{\xi}\left(\theta^{*}(r)\right)$ are given in Theorem 2.3.

Proof Choose $u, v \in[0, T]$ such that $v \geq u$. Then the discounted stock price is given by $S^{*}(v):=S(v) e^{-\int_{u}^{v} r(s) \mathrm{d} s}$. Using this and a version of the Bayes's rule, we get

$$
\begin{aligned}
& E^{\mathbb{Q}_{\theta^{*}}}\left[S^{*}(v) \mid \mathcal{G}_{u}\right]=S^{*}(u) E^{\mathbb{Q}_{\theta^{*}}}\left[e^{-\int_{u}^{v} r(s) \mathrm{d} s} e^{\int_{u}^{v} \mathrm{~d} Y(s)} \mid \mathcal{G}_{u}\right] \\
& =S^{*}(u) \frac{E^{\mathbb{P}}\left[e^{-\int_{u}^{v} r(s) \mathrm{d} s} e^{\int_{u}^{v} \mathrm{~d} Y(s)} \Lambda^{\theta^{*}}(v) \mid \mathcal{G}_{u}\right]}{E^{\mathbb{P}}\left[\Lambda^{\theta^{*}}(v) \mid \mathcal{G}_{u}\right]} \\
& =S^{*}(u) \frac{E^{\mathbb{P}}\left[e^{-\int_{u}^{v} r(s) \mathrm{d} s} e^{\int_{u}^{v} \mathrm{~d} Y(s)} \Lambda^{\theta^{*}}(v) \mid \mathcal{G}_{u}\right]}{E^{\mathbb{P}}\left[\Lambda^{\theta^{*}}(v) \mid \mathcal{G}_{u}\right]} \\
& =S^{*}(u) \frac{E^{\mathbb{P}}\left[e^{-\int_{u}^{v} r(s) \mathrm{d} s} e^{-\int_{u}^{v}\left(\theta^{*}(s)-1\right) \mathrm{d} Y(s)} E^{\mathbb{P}}\left[e^{-\int_{v}^{T} \theta^{*}(s) \mathrm{d} Y(s)} \mid \mathcal{G}_{v}\right] \mid \mathcal{G}_{u}\right]}{E^{\mathbb{P}}\left[e^{-\int_{u}^{T} \theta^{*}(s) \mathrm{d} Y(s)} \mid \mathcal{G}_{u}\right]}
\end{aligned}
$$


Using the occupation times (Lemma 2.2.)

$$
\begin{aligned}
E^{\mathbb{Q}_{\theta^{*}}} & {\left[S^{*}(v) \mid \mathcal{G}_{u}\right] } \\
= & S^{*}(u) \frac{E^{\mathbb{P}}\left[\exp \left(\sum_{i=1}^{N} \int_{u}^{v} \tilde{\xi}_{i}\left(\theta_{i}^{*}(t)\right) \mathrm{d} J_{i}(u, t)\right) E^{\mathbb{P}}\left[\exp \left(\sum_{i=1}^{N} \int_{v}^{T} \xi_{i}\left(\theta_{i}^{*}(t)\right) \mathrm{d} J_{i}(v, t)\right) \mid \mathcal{G}_{v}\right] \mid \mathcal{G}_{u}\right]}{} \\
& E^{\mathbb{P}}\left[\exp \left(\sum_{i=1}^{N} \int_{u}^{v} \xi_{i}\left(\theta_{i}^{*}(t)\right) \mathrm{d} J_{i}(u, t)\right) E^{\mathbb{P}}\left[\exp \left(\sum_{i=1}^{N} \int_{v}^{T} \xi_{i}\left(\theta_{i}^{*}(t)\right) \mathrm{d} J_{i}(v, t)\right)\left|\mathcal{G}_{v}\right| \mathcal{G}_{u}\right] .\right.
\end{aligned}
$$

Using the following property of homogeneous Markov chains

$$
\begin{aligned}
\operatorname{Law}\left(J_{1}(v, T), \ldots, J_{N}(v, T) \mid \mathcal{G}(v)\right) & =\operatorname{Law}\left(J_{1}(v, T), \ldots, J_{N}(v, T) \mid X(v)\right) \\
& =\operatorname{Law}\left(J_{1}(0, T-v), \ldots, J_{N}(0, T-v) \mid X(0)\right)
\end{aligned}
$$

(2.28) becomes

$$
\begin{aligned}
& E^{\mathbb{Q}_{\theta^{*}}}\left[S^{*}(v) \mid \mathcal{G}_{u}\right] \\
& =S^{*}(u) \frac{E^{\mathbb{P}}\left[\exp \left(\sum_{i=1}^{N} \int_{0}^{T-v} \xi_{i}\left(\theta_{i}^{*}(t)\right) \mathrm{d} J_{i}(0, t)\right) \mid X(0)\right] E^{\mathbb{P}}\left[\exp \left(\sum_{i=1}^{N} \int_{u}^{v} \tilde{\xi}_{i}\left(\theta_{i}^{*}(t)\right) \mathrm{d} J_{i}(u, t)\right) \mid \mathcal{G}_{u}\right]}{\left.E^{\mathbb{P}}\left[\exp \left(\sum_{i=1}^{N} \int_{0}^{T-v} \xi_{i}\left(\theta_{i}^{*}(t)\right) \mathrm{d} J_{i}(0, t)\right\rangle\right) \mid X(0)\right] E^{\mathbb{P}}\left[\exp \left(\sum_{i=1}^{N} \int_{u}^{v} \xi_{i}\left(\theta_{i}^{*}(t)\right) \mathrm{d} J_{i}(u, t)\right) \mid \mathcal{G}_{u}\right]} .
\end{aligned}
$$

This implies

$$
E^{\mathbb{Q}_{\theta^{*}}}\left[S^{*}(v) \mid \mathcal{G}_{u}\right]=S^{*}(u) \frac{E^{\mathbb{P}}\left[\exp \left(\sum_{i=1}^{N} \int_{u}^{v} \tilde{\xi}_{i}\left(\theta_{i}^{*}(t)\right) \mathrm{d} J_{i}(u, t)\right) \mid \mathcal{G}_{u}\right]}{E^{\mathbb{P}}\left[\exp \left(\sum_{i=1}^{N} \int_{u}^{v} \xi_{i}\left(\theta_{i}^{*}(t)\right) \mathrm{d} J_{i}(u, t)\right) \mid \mathcal{G}_{u}\right]} .
$$

Hence, using Lemma 2.2, we get

$$
E^{\mathbb{Q}_{\theta^{*}}}\left[S^{*}(v) \mid \mathcal{G}_{u}\right]=S^{*}(u) \frac{\left\langle e^{\int_{u}^{v}\left(\mathbf{A}+\operatorname{Diag}\left(\underline{\tilde{\xi}}\left(\theta^{*}(r)\right)\right) \mathrm{d} r\right.} X(u), \underline{\mathbf{1}}\right\rangle}{\left\langle e^{\int_{u}^{v}\left(\mathbf{A}+\operatorname{Diag}\left(\underline{\xi}\left(\theta^{*}(r)\right)\right)\right) \mathrm{d} r} X(u), \underline{\mathbf{1}}\right\rangle} .
$$

Proof of Theorem 2.3 This follows directly from the previous lemma by setting $v=t$ and $u=0$ in (2.26). In fact, we have that the martingale condition (2.19) is equivalent to $(2.23)$.

We turn our main focus on the condition for the family $\left\{\mathbb{Q}_{\theta^{*}}: \theta^{*} \in \Theta^{*}\right\}$ because through a standard approximation for the matrix exponential in (2.23), we shall deduce the martingale condition for the family $\left\{\mathbb{Q}^{\theta}: \theta \in \Theta\right\}$; See Siu and Yang (2009); Momeya and Morales (2014).

\subsection{Approximations}

Here, we analyse the two families of equivalent martingale measures $\mathbb{Q}^{\theta}$ and $\mathbb{Q}_{\theta^{*}}$ via certain types of approximations for the martingale condition (2.23). The exponential of a $N \times N$ matrix $\mathbf{E}$ is defined as 


$$
\exp (\mathbf{E}):=\sum_{n=0}^{\infty} \frac{E^{n}}{n !}
$$

where $\mathbf{E}^{0}=\mathbf{I}$ is the identity matrix and by convention $0 !=1$. Replacing $X(0)$ by $\mathbf{e}_{i}$ for $i=1, \ldots, N$ in (2.23) yields,

$$
\left\langle e^{\int_{0}^{t}\left(\mathbf{A}+\operatorname{Diag}\left(\underline{\tilde{\xi}}\left(\theta^{*}(r)\right)\right)\right) \mathrm{d} r} \mathbf{e}_{i}, \underline{\mathbf{1}}\right\rangle-\left\langle e^{\int_{0}^{t}\left(\mathbf{A}+\operatorname{Diag}\left(\underline{\xi}\left(\theta^{*}(r)\right)\right)\right) \mathrm{d} r} \mathbf{e}_{i}, \underline{\mathbf{1}}\right\rangle=0 .
$$

This is a system of $N$ equations and in practice, to solve it, one needs to adopt a finite number of terms in the series' expansion of $\exp (\mathbf{E})$. Using the first-order approximation of $\exp (\mathbf{E})$ (i.e., $\exp (\mathbf{E}) \approx \mathbf{I}+\mathbf{E})$ in $(2.31)$, we have

$$
\begin{aligned}
& \left\langle\left(\mathbf{I}+\int_{0}^{t}\left(\mathbf{A}+\operatorname{Diag}\left(\underline{\tilde{\xi}}\left(\theta^{*}(r)\right)\right)\right) \mathrm{d} r\right) \mathbf{e}_{i}, \underline{\mathbf{1}}\right\rangle \\
& \quad-\left\langle\left(\mathbf{I}+\int_{0}^{t}\left(\mathbf{A}+\operatorname{Diag}\left(\underline{\xi}\left(\theta^{*}(r)\right)\right)\right) \mathrm{d} r\right) \mathbf{e}_{i}, \underline{\mathbf{1}}\right\rangle=0 .
\end{aligned}
$$

This yields

$$
\begin{aligned}
& \left(\sum_{k=1, k \neq i}^{N} t a_{k i}+1+a_{i i} t+\int_{0}^{t} \tilde{\xi}_{i}\left(\theta_{i}^{*}(r)\right) \mathrm{d} r\right) \\
& -\left(\sum_{k=1, k \neq i}^{N} t a_{k i}+1+a_{i i} t+\int_{0}^{t} \xi_{i}\left(\theta_{i}^{*}(r)\right) \mathrm{d} r\right)=0,
\end{aligned}
$$

i.e.,

$$
\int_{0}^{t} \tilde{\xi}_{i}\left(\theta_{i}^{*}(r)\right) \mathrm{d} r-\int_{0}^{t} \xi_{i}\left(\theta_{i}^{*}(r)\right) \mathrm{d} r=0, \text { for } i=1,2, \ldots, N,
$$

which simplifies to

$\mu_{i}(t)-r_{i}(t)-\theta_{i}(t) \sigma_{i}^{2}(t)+\int_{\mathbb{R}}\left(e^{z}-1\right)\left(e^{-z \theta_{i}(t)}-1\right) \rho_{i}(z) \mathrm{d} z=0, t$-a.e., $\forall t \in[0, T]$.

Eq. (2.32) coincides with the martingale condition for the family $\left\{\mathbb{Q}^{\theta}: \theta \in \Theta\right\}$ as given in (2.20). Hence, the martingale condition for the family $\left\{\mathbb{Q}^{\theta}: \theta \in \Theta\right\}$ is a first order approximation of the martingale condition for $\left\{\mathbb{Q}_{\theta^{*}}: \theta^{*} \in \Theta^{*}\right\}$. We can think of the pricing kernel $\Lambda^{\theta^{*}}$ as having more information than the kernel $\Lambda^{\theta}$ with $\theta^{*}$ been more realistic.

We will now as in Siu and Yang (2009); Momeya and Morales (2014) derive the martingale condition for $\mathbb{Q}_{\theta^{*}}$ by taking a two-order approximation for the matrix exponential in (2.30). This will enable to move from the less realistic assumption where the whole path of the Markov chain is known to the more realistic one where 
only the initial state in know. The approximation is given by

$$
\exp (\mathbf{E}) \approx \mathbf{I}+\mathbf{E}+\frac{1}{2} \mathbf{E}^{2}
$$

For simplicity, we consider two regimes i.e, $N=2$ and we set $a_{11}=-a_{12}=-a$ and $a_{21}=-a_{22}=a ; a \geq 0$ and $t>0$. In this case, we need to solve the following pair of equations:

$$
\begin{aligned}
& \left\langle e^{\int_{0}^{t}\left(\mathbf{A}+\operatorname{Diag}\left(\underline{\tilde{\xi}}\left(\theta^{*}(r)\right)\right)\right) \mathrm{d} r} \mathbf{e}_{1}, \underline{\mathbf{1}}\right\rangle-\left\langle e^{\int_{0}^{t}\left(\mathbf{A}+\operatorname{Diag}\left(\underline{\xi}\left(\theta^{*}(r)\right)\right)\right) \mathrm{d} r} \mathbf{e}_{1}, \underline{\mathbf{1}}\right\rangle=0, \\
& \left\langle e^{\int_{0}^{t}\left(\mathbf{A}+\operatorname{Diag}\left(\underline{\tilde{\xi}}\left(\theta^{*}(r)\right)\right)\right) \mathrm{d} r} \mathbf{e}_{2}, \underline{\mathbf{1}}\right\rangle-\left\langle e^{\int_{0}^{t}\left(\mathbf{A}+\operatorname{Diag}\left(\underline{\xi}\left(\theta^{*}(r)\right)\right)\right) \mathrm{d} r} \mathbf{e}_{2}, \underline{\mathbf{1}}\right\rangle=0
\end{aligned}
$$

for $\underline{\mathbf{1}}=(1,1)^{\prime} \in \mathbb{R}^{2}$. But

$$
\left.E=\int_{0}^{t}\left(\mathbf{A}+\operatorname{Diag}\left(\underline{\tilde{\xi}}\left(\theta^{*}(r)\right)\right)\right) \mathrm{d} r\right)
$$

or

$$
E=\left(\begin{array}{cc}
\int_{0}^{t}\left(-a+\tilde{\xi}_{1}\left(\theta_{1}^{*}(r)\right)\right) \mathrm{d} r & a t \\
a t & \int_{0}^{t}\left(-a+\tilde{\xi}_{2}\left(\theta_{2}^{*}(r)\right)\right) \mathrm{d} r
\end{array}\right)
$$

Substituting (2.33) in (2.34), the martingale condition (2.23), for $X(0)=\mathbf{e}_{1}=(1,0)^{\prime}$ becomes

$$
\begin{aligned}
& \int_{0}^{t}\left(\tilde{\xi}_{1}\left(\theta_{1}^{*}(r)\right)-\xi_{1}\left(\theta_{1}^{*}(r)\right)\right) \mathrm{d} r-a t \int_{0}^{t}\left(\tilde{\xi}_{1}\left(\theta_{1}^{*}(r)\right)-\xi_{1}\left(\theta_{1}^{*}(r)\right)\right) \mathrm{d} r \\
& +\frac{1}{2}\left\{\left[\int_{0}^{t}\left(\tilde{\xi}_{1}\left(\theta_{1}^{*}(r)\right)-\xi_{1}\left(\theta_{1}^{*}(r)\right)\right) \mathrm{d} r\right]\left[\int_{0}^{t}\left(\tilde{\xi}_{1}\left(\theta_{1}^{*}(r)\right)+\xi_{1}\left(\theta_{1}^{*}(r)\right)\right) \mathrm{d} r\right]\right. \\
& \left.+a t \int_{0}^{t}\left(\tilde{\xi}_{2}\left(\theta_{2}^{*}(r)\right)-\xi_{2}\left(\theta_{2}^{*}(r)\right)\right) \mathrm{d} r\right\}=0
\end{aligned}
$$

Similarly, for $X(0)=\mathbf{e}_{2}=(0,1)$, substituting (2.33) in (2.35), we get

$$
\begin{aligned}
& \int_{0}^{t}\left(\tilde{\xi}_{2}\left(\theta_{2}^{*}(r)\right)-\xi_{2}\left(\theta_{2}^{*}(r)\right)\right) \mathrm{d} r-a t \int_{0}^{t}\left(\tilde{\xi}_{2}\left(\theta_{2}^{*}(r)\right)-\xi_{2}\left(\theta_{2}^{*}(r)\right)\right) \mathrm{d} r \\
& +\frac{1}{2}\left\{\left[\int_{0}^{t}\left(\tilde{\xi}_{2}\left(\theta_{2}^{*}(r)\right)-\xi_{2}\left(\theta_{2}^{*}(r)\right)\right) \mathrm{d} r\right]\left[\int_{0}^{t}\left(\tilde{\xi}_{2}\left(\theta_{2}^{*}(r)\right)+\xi_{2}\left(\theta_{2}^{*}(r)\right)\right) \mathrm{d} r\right]\right. \\
& \left.+a t \int_{0}^{t}\left(\tilde{\xi}_{1}\left(\theta_{1}^{*}(r)\right)-\xi_{1}\left(\theta_{1}^{*}(r)\right)\right) \mathrm{d} r\right\}=0 .
\end{aligned}
$$


Here

$$
\begin{aligned}
\tilde{\xi}_{i}\left(\theta_{i}^{*}(t)\right)-\xi_{i}\left(\theta_{i}^{*}(t)\right)= & \mu_{i}(t)-r_{i}(t)-\theta_{i}^{*}(t) \sigma_{i}^{2}(t) \\
& +\int_{\mathbb{R}}\left(e^{z}-1\right)\left(e^{-z \theta_{i}^{*}(t)}-1\right) \rho_{i}(z) \mathrm{d} z, t \text {-a.e }
\end{aligned}
$$

and

$$
\begin{aligned}
\tilde{\xi}_{i}\left(\theta_{i}^{*}(t)\right)+\xi_{i}\left(\theta_{i}^{*}(t)\right)= & \mu_{i}(t)-r_{i}(t)-2 \theta_{i}^{*}(t) \mu_{i}(t)+\left(\theta_{i}^{*}(t)\right)^{2} \sigma_{i}^{2}(t) \\
& +\int_{\mathbb{R}}\left(e^{-z\left(\theta_{i}^{*}(t)-1\right)}+e^{-z \theta_{i}^{*}(t)}-2\right) \\
& +\left(2 \theta_{i}^{*}(t)-1\right)\left(e^{-z}-1\right) \rho_{i}(z) \mathrm{d} z, t \text {-a.e. }
\end{aligned}
$$

for $i=1,2$. (2.38) and (2.39) are more tractable than (2.23) and we shall use them to determine the EMM parameters $\left(\theta_{1}^{*}(t), \theta_{2}^{*}(t)\right)$ for the numerical illustrations.

\subsection{Particular Cases}

In this section, we present the developments made in the previous section for particular models. In the sequel, we take $N=2$, i.e., the Markov chain $X$ moves only between the two states $\mathbf{e}_{1}=(1,0)^{T}$ and $\mathbf{e}_{2}=(0,1)^{T}$. We shall give explicit martingale conditions for regime-switching Black-Scholes, Variance Gamma (VG) and Carr Geman Madan and Yor (CGMY) models when the coefficient are constants. Note that the former cases of regime-switching Black-Scholes and Variance Gamma models were already derived in Siu and Yang (2009) and Momeya and Morales (2014).

\subsubsection{The Regime-Switching Black-Scholes Model}

In this section, we present the regime switching Black-Scholes model. The dynamic of price of the risky asset in this case is given by

$$
S(t)=S(0) \exp \left\{\int_{0}^{t}\left(\mu(s)-\frac{1}{2} \sigma^{2}(s)\right) \mathrm{d} s+\int_{0}^{t} \sigma(s) \mathrm{d} W(s)\right\} .
$$

In the following theorem, we give (without proof) the equation satisfied by the state price density $\theta_{i}$ and $\theta_{i}^{*}$.

Theorem 2.5 Assume that the dynamic of the stock price is given by (2.42). Then the values of $\theta_{i}$ satisfying the martingale condition (2.20) are reduced to

$$
\theta_{i}=\frac{\mu_{i}-r_{i}}{\sigma_{i}^{2}} \text { for } i=1,2
$$


Moreover, $\theta_{i}^{*}$ in (2.23) satisfy the following system of nonlinear equations in $\left(\theta_{1}^{*}, \theta_{2}^{*}\right)$,

$$
\begin{gathered}
\frac{\sigma_{1}^{4} t^{2}}{2}\left(\theta_{1}^{*}\right)^{3}-\frac{\left(3 \mu_{1}-r_{1}\right) \sigma_{1}^{2} t^{2}}{2}\left(\theta_{1}^{*}\right)^{2}+\left(\sigma_{1}^{2} t+\frac{\left(\mu_{1}-r_{1}\right)\left(\sigma_{1}^{2}(t)+2 \mu_{1}\right) t^{2}-a \sigma_{1}^{2} t^{2}}{2}\right) \theta_{1}^{*}(t) \\
+\frac{a \sigma_{2}^{2} t^{2}}{2} \theta_{2}^{*}-\left(\frac{\left(\mu_{1}-r_{1}\right)^{2}-a\left(\mu_{1}-r_{1}\right)+a\left(\mu_{2}-r_{2}\right)}{2}\right) t^{2}-\left(\mu_{1}-r_{1}\right) t=0, t \in[0, T]
\end{gathered}
$$

and

$$
\begin{aligned}
& \frac{\sigma_{2}{ }^{4} t^{2}}{2}\left(\theta_{2}^{*}\right)^{3}-\frac{\left(3 \mu_{2}-r_{2}\right) \sigma_{2}{ }^{2} t^{2}}{2}\left(\theta_{2}^{*}\right)^{2} \\
& \quad+\left(\sigma_{2}{ }^{2} t+\frac{\left(\mu_{2}-r_{2}\right)\left(\sigma_{2}{ }^{2}+2 \mu_{2}\right) t^{2}-a \sigma_{2}{ }^{2} t^{2}}{2}\right) \theta_{2}^{*}+\frac{a \sigma_{1}{ }^{2} t^{2}}{2} \theta_{1}^{*} \\
& \quad-\left(\frac{\left(\mu_{2}-r_{2}\right)^{2}-a\left(\mu_{2}-r_{2}\right)+a\left(\mu_{1}-r_{1}\right)}{2}\right) t^{2}-\left(\mu_{2}-r_{2}\right) t=0, t \in[0, T] .
\end{aligned}
$$

Proof See Siu and Yang (2009).

\subsubsection{The Regime-Switching Variance-Gamma Model}

In this section we present the regime switching variance-gamma model. We obtain this model from the general model for the risky asset described in Eq. (2.6) by setting the dynamics of the process as

$$
\begin{aligned}
S(t)= & S(0) \exp \left[\int_{0}^{t} \mu(s) \mathrm{d} s+\int_{0}^{t} \int_{\mathbb{R} \backslash\{0\}} z \tilde{N}_{V G}^{X}(\mathrm{~d} s, \mathrm{~d} z)\right. \\
& \left.-\int_{0}^{t} \int_{\mathbb{R} \backslash\{0\}}\left(e^{z}-1-z\right) \rho_{V G}^{X}(\mathrm{~d} z) \mathrm{d} s\right],
\end{aligned}
$$

where the jump process $N_{V G}(\cdot, \cdot)$ has the predictable compensator

$$
\rho_{V G}^{X}(\mathrm{~d} z) \mathrm{d} t=\sum_{i=1}^{2}\left\langle\mathbf{e}_{i}, X\left(t^{-}\right)\right\rangle \rho_{i}^{V G}(z) \mathrm{d} t,
$$

with the Lévy measure associated to the variance gamma process as

$$
\rho_{i}^{V G}(z)=C_{i} \frac{e^{-G_{i}|z|}}{|z|} 1_{z<0}+C_{i} \frac{e^{-M_{i}|z|}}{|z|} 1_{z>0}
$$

We then have the following martingale conditions theorem 
Theorem 2.6 Assume that the dynamic of the stock price is given by (2.46). Then the values of $\theta_{i}$ satisfying the martingale condition (2.20) are reduced to

$\mu_{i}-r_{i}-C_{i} \log \left(\frac{G_{i} M_{i}}{\left(G_{i}+1\right)\left(M_{i}-1\right)}\right)+C_{i} \log \left(\frac{\left(G_{i}-\theta_{i}\right)\left(M_{i}+\theta_{i}\right)}{\left(G_{i}-\theta_{i}+1\right)\left(M_{i}+\theta_{i}-1\right)}\right)=0$

for $i=1,2$. Moreover, $\theta_{i}^{*}$ in (2.23) satisfy the following system of nonlinear equations in $\left(\theta_{1}^{*}, \theta_{2}^{*}\right)$

$$
\begin{aligned}
& \left\{\mu_{1}-r_{1}-C_{1} \log \left(\frac{G_{1} M_{1}}{\left(G_{1}+1\right)\left(M_{1}-1\right)}\right)+C_{1} \log \left(\frac{\left(G_{1}-\theta_{1}^{*}\right)\left(M_{1}+\theta_{1}^{*}\right)}{\left(G_{1}-\theta_{1}^{*}+1\right)\left(M_{1}+\theta_{1}^{*}-1\right)}\right)\right\} \\
& \quad \times\left\{t+\frac{1}{2} t^{2}\left[\mu_{1}-r_{1}-2 \theta_{1}^{*} \mu_{1}+C_{1} \log \left(\frac{G_{1} M_{1}}{\left(G_{1}-\theta_{1}^{*}\right)\left(M_{1}+\theta_{1}^{*}\right)}\right)\right.\right. \\
& +C_{1} \log \left(\frac{G_{1} M_{1}}{\left(G_{1}-\theta_{1}^{*}+1\right)\left(M_{1}+\theta_{1}^{*}-1\right)}\right)+\left(2 \theta_{1}^{*}-1\right) C_{1} \\
& \left.\left.\times \log \left(\frac{G_{1} M_{1}}{\left(G_{1}+1\right)\left(M_{1}-1\right)}\right)\right]-a\right\}+\frac{1}{2} a t^{2}\left\{\mu_{2}-r_{2}-C_{2} \log \left(\frac{G_{2} M_{2}}{\left(G_{2}+1\right)\left(M_{2}-1\right)}\right)\right. \\
& \left.+C_{2} \log \left(\frac{\left(G_{2}-\theta_{2}^{*}\right)\left(M_{2}+\theta_{2}^{*}\right)}{\left(G_{2}-\theta_{2}^{*}+1\right)\left(M_{2}+\theta_{2}^{*}-1\right)}\right)\right\}=0
\end{aligned}
$$

and

$$
\begin{aligned}
& \left\{\mu_{2}-r_{2}-C_{2} \log \left(\frac{G_{2} M_{2}}{\left(G_{2}+1\right)\left(M_{2}-1\right)}\right)+C_{2} \log \left(\frac{\left(G_{2}-\theta_{2}^{*}\right)\left(M_{2}+\theta_{2}^{*}\right)}{\left(G_{2}-\theta_{2}^{*}+1\right)\left(M_{2}+\theta_{2}^{*}-1\right)}\right)\right\} \\
& \quad \times\left\{t+\frac{1}{2} t^{2}\left[\mu_{2}-r_{2}-2 \theta_{2}^{*} \mu_{2}+C_{2} \log \left(\frac{G_{2} M_{2}}{\left(G_{2}-\theta_{2}^{*}\right)\left(M_{2}+\theta_{2}^{*}\right)}\right)\right.\right. \\
& \quad+C_{2} \log \left(\frac{G_{2} M_{2}}{\left(G_{2}-\theta_{2}^{*}+1\right)\left(M_{2}+\theta_{2}^{*}-1\right)}\right)+\left(2 \theta_{2}^{*}-1\right) C_{2} \\
& \left.\left.\quad \times \log \left(\frac{G_{2} M_{2}}{\left(G_{2}+1\right)\left(M_{2}-1\right)}\right)\right]-a\right\}+\frac{1}{2} a t^{2}\left\{\mu_{1}-r_{1}-C_{1} \log \left(\frac{G_{1} M_{1}}{\left(G_{1}+1\right)\left(M_{1}-1\right)}\right)\right. \\
& \left.\quad+C_{1} \log \left(\frac{\left(G_{1}-\theta_{1}^{*}\right)\left(M_{1}+\theta_{1}^{*}\right)}{\left(G_{1}-\theta_{1}^{*}+1\right)\left(M_{1}+\theta_{1}^{*}-1\right)}\right)\right\}=0 .
\end{aligned}
$$

Proof See Momeya and Morales (2014). 


\subsubsection{The regime-Switching CGMY Model}

In this section we present the regime switching CGMY. This model is obtained from the general case by setting the dynamics of the risky process $S$ as

$$
\begin{aligned}
S(t)= & S(0) \exp \left[\int_{0}^{t} \mu(s) \mathrm{d} s+\int_{0}^{t} \int_{\mathbb{R} \backslash\{0\}} z \tilde{N}_{C G M Y}^{X}(\mathrm{~d} s, \mathrm{~d} z)\right. \\
& \left.-\int_{0}^{t} \int_{\mathbb{R} \backslash\{0\}}\left(e^{z}-1-z\right) \rho_{C G M Y}^{X}(\mathrm{~d} z) \mathrm{d} s\right]
\end{aligned}
$$

where the jump process $N_{C G M Y}^{X}(t ; \cdot)$ has the predictable compensator

$$
\rho_{C G M Y}^{X}(\mathrm{~d} z) \mathrm{d} t=\sum_{i=1}^{2}\left\langle\mathbf{e}_{i}, X\left(t^{-}\right)\right\rangle \rho_{i}^{C G M Y}(z) \mathrm{d} t
$$

with the Lévy measure associated to the CGMY process as

$$
\rho_{i}^{C G M Y}(z)=C_{i} \frac{e^{-G_{i}|z|}}{|z|^{1+Y}} 1_{z<0}+C_{i} \frac{e^{-M_{i}|z|}}{|z|^{1+Y}} 1_{z>0} .
$$

In the following theorem, we derive the equation satisfied by the state price density $\theta_{i}$ of the equivalent martingale measure $\mathbb{Q}^{\theta_{i}}$ when the price of risk in the regime switching model is not taken into account.

Theorem 2.7 (Martingale condition without price of risk) Assume that the dynamic of the stock price is given by (2.52). Moreover assume that the state price density $\theta_{i}$ is such that $0<\theta_{i}<G_{i}$ and $M_{i}>1$. Then $\theta_{i}(t)$ satisfies the following system of equations

$$
\begin{aligned}
& \mu_{i}-r_{i}+C_{i} \Gamma\left(-Y_{i}\right)\left[\left(G_{i}-\left(\theta_{i}-1\right)\right)^{Y_{i}}-\left(G_{i}+1\right)^{Y_{i}}-\left(G_{i}-\theta_{i}\right)^{Y_{i}}\right. \\
& \left.\quad+G_{i}^{Y_{i}}+M_{i}^{Y_{i}}+\left(M_{i}+\theta_{i}-1\right)^{Y_{i}}-\left(M_{i}-1\right)^{Y_{i}}-\left(M_{i}+\theta_{i}\right)^{Y_{i}}\right]=0 \\
& \text { for } i=1,2 .
\end{aligned}
$$

Proof Assume that $S$ satisfies (2.52), then (2.20) is reduced to

$$
\mu_{i}-r_{i}+\int_{\mathbb{R}}\left(e^{z}-1\right)\left(e^{-z \theta_{i}}-1\right) v_{i}(z) \mathrm{d} z=0, \quad i=1,2
$$


The integral term involved in Eq. (2.56) is computed as follows

$$
\begin{aligned}
\int_{\mathbb{R}}\left(e^{z}-1\right)\left(e^{-z \theta_{i}}-1\right) \nu_{i}(z) \mathrm{d} z= & \int_{\mathbb{R}}\left(e^{z}-1\right)\left(e^{-z \theta_{i}}-1\right) \\
& \times\left(C_{i} \frac{e^{-G_{i}|z|}}{|z|^{1+Y}} 1_{z<0}+C_{i} \frac{e^{-M_{i}|z|}}{|z|^{1+Y}} 1_{z>0}\right) \mathrm{d} z \\
= & \int_{-\infty}^{0}\left(e^{z}-1\right)\left(e^{-z \theta_{i}}-1\right) \frac{C_{i} \exp \left(G_{i} z\right)}{(-z)^{Y_{i}+1}} \mathrm{~d} z \\
& +\int_{0}^{\infty}\left(e^{z}-1\right)\left(e^{-z \theta_{i}}-1\right) \frac{C_{i} \exp \left(-M_{i} z\right)}{(z)^{Y_{i}+1}} \mathrm{~d} z \\
= & I_{1}+I_{2} .
\end{aligned}
$$

We shall now consider different cases

Case 1: $\quad Y=0$.

This is the variance gamma case and was discussed in the previous section.

Case 2: $\quad Y \neq 0$. We have that

$$
\begin{aligned}
I_{1}= & \int_{-\infty}^{0}\left(e^{-z\left(\theta_{i}-1\right)}-e^{z}-e^{-z \theta_{i}}+1\right) \frac{C_{i} e^{G_{i} z}}{(-z)^{Y_{i}+1}} \mathrm{~d} z \\
= & C_{i}\left[\int_{-\infty}^{0}\left(e^{G_{i}-z\left(\theta_{i}-1\right)}(-z)^{-1-Y_{i}}\right) \mathrm{d} z-\int_{-\infty}^{0}\left(e^{\left(G_{i}+1\right) z}(-z)^{-1-Y_{i}}\right) \mathrm{d} z\right. \\
& \left.-\int_{-\infty}^{0}\left(e^{\left(G_{i}-\theta_{i}\right) z}(-z)^{-1-Y_{i}}\right) \mathrm{d} z-\int_{-\infty}^{0}\left(e^{G_{i} z}(-z)^{-1-Y_{i}}\right) \mathrm{d} z\right]
\end{aligned}
$$

Put $w=-\left(G_{i}-\left(\theta_{i}-1\right)\right) z, w=-\left(G_{i}+1\right) z, w=-\left(G_{i}-\theta_{i}\right) z, w=-G_{i} z$ in the first, second, third and fourth integral respectively, then using the definition of the gamma function, we get

$$
I_{1}=C_{i} \Gamma\left(-Y_{i}\right)\left[\left(G_{i}-\left(\theta_{i}-1\right)\right)^{Y_{i}}-\left(G_{i}+1\right)^{Y_{i}}-\left(G_{i}-\theta_{i}\right)^{Y_{i}}+G^{Y_{i}}\right]
$$

In the same way, $I_{2}$ is solved explicitly using change of variable and the definition of the gamma function to get

$$
I_{2}=C_{i} \Gamma\left(-Y_{i}\right)\left[\left(M_{i}+\theta_{i}-1\right)^{Y_{i}}-\left(M_{i}-1\right)^{Y_{i}}-\left(M_{i}+\theta_{i}\right)^{Y_{i}}+M_{i}^{Y_{i}}\right] .
$$


Combining (2.59) and (2.60), we get

$$
\begin{aligned}
\int_{\mathbb{R}}\left(e^{z}-1\right)\left(e^{-z \theta_{i}}-1\right) \rho_{i}(z) \mathrm{d} z= & C_{i} \Gamma\left(-Y_{i}\right)\left[\left(G_{i}-\left(\theta_{i}-1\right)\right)^{Y_{i}}-\left(G_{i}+1\right)^{Y_{i}}\right. \\
& +G_{i}^{Y_{i}}-\left(G_{i}-\theta_{i}\right)^{Y_{i}}+\left(M_{i}+\theta_{i}-1\right)^{Y_{i}} \\
& \left.-\left(M_{i}-1\right)^{Y_{i}}-\left(M_{i}+\theta_{i}\right)^{Y_{i}}+M_{i}^{Y_{i}}\right]
\end{aligned}
$$

Substituting this into Eq. (2.56) gives us the desired result.

In the following theorem, we derive the equation satisfied by the state price density $\theta_{i}^{*}$ of the equivalent martingale measure $\mathbb{Q}_{\theta_{i}^{*}}$ when the price of risk in the regime switching model is taken into account.

Theorem 2.8 (Martingale condition with price of risk) Assume that conditions of Theorem (2.7) are satisfied. Then the state price densities $\theta_{i}^{*}(t)$ in (2.23) satisfy the following system of non linear equations in $\left.\left(\theta_{1}^{*}, \theta_{2}^{*}\right)\right)$,

$$
\begin{aligned}
& \left\{\mu_{1}-r_{1}+C_{1} \Gamma\left(-Y_{1}\right)\left[\left(G_{1}-\left(\theta_{1}-1\right)\right)^{Y_{1}}-\left(G_{1}+1\right)^{Y_{1}}-\left(G_{1}-\theta_{1}\right)^{Y_{1}}\right.\right. \\
& \left.\left.\quad+G_{1}^{Y_{1}}+M_{1}^{Y_{1}}+\left(M_{1}+\theta_{1}-1\right)^{Y_{1}}-\left(M_{1}-1\right)^{Y_{1}}-\left(M_{1}+\theta_{1}\right)^{Y_{1}}\right]\right\} \\
& \quad \times\left\{t+\frac{1}{2} t^{2}\left(\mu_{1}-r_{1}-2 \theta_{1}^{*} \mu_{1}+C_{1} \Gamma\left(-Y_{1}\right)\left[\left(G_{1}-\left(\theta_{1}^{*}-1\right)\right)^{Y_{1}}\right.\right.\right. \\
& \quad+\left(G_{1}-\theta_{1}^{*}\right)^{Y_{1}}+\left(2 \theta_{1}^{*}-1\right)\left(G_{1}+1\right)^{Y_{1}}-\left(2 \theta_{1}^{*}+1\right) G_{1}^{Y_{1}}+\left(M_{1}+\theta_{1}^{*}-1\right)^{Y_{1}} \\
& \left.\left.\left.\quad+\left(M_{1}+\theta_{1}\right)^{Y_{1}}+\left(2 \theta_{1}^{*}-1\right)\left(M_{1}-1\right)^{Y_{1}}-\left(2 \theta_{1}^{*}+1\right) M_{1}^{Y_{1}}\right]\right)-a\right\} \\
& \quad+\frac{1}{2} t^{2} a\left\{\mu_{2}-r_{2}+C_{2} \Gamma\left(-Y_{2}\right)\left[\left(G_{2}-\left(\theta_{2}-1\right)\right)^{Y_{2}}-\left(G_{2}+1\right)^{Y_{2}}+G_{2}^{Y_{2}}+M_{2}^{Y_{2}}\right.\right. \\
& \left.\left.\quad-\left(G_{2}-\theta_{2}\right)^{Y_{2}}+\left(M_{2}+\theta_{2}-1\right)^{Y_{2}}-\left(M_{2}-1\right)^{Y_{2}}-\left(M_{2}+\theta_{2}\right)^{Y_{2}}\right]\right\}=0
\end{aligned}
$$

and

$$
\begin{aligned}
& \left\{\mu_{2}-r_{2}+C_{2} \Gamma\left(-Y_{2}\right)\left[\left(G_{2}-\left(\theta_{2}-1\right)\right)^{Y_{2}}-\left(G_{2}+1\right)^{Y_{2}}-\left(G_{2}-\theta_{2}\right)^{Y_{2}}\right.\right. \\
& \left.\left.\quad+G_{2}^{Y_{2}}+M_{2}^{Y_{2}}+\left(M_{2}+\theta_{2}-1\right)^{Y_{2}}-\left(M_{2}-1\right)^{Y_{2}}-\left(M_{2}+\theta_{2}\right)^{Y_{2}}\right]\right\} \\
& \quad \times\left\{t+\frac{1}{2} t^{2}\left(\mu_{2}-r_{2}-2 \theta_{2}^{*} \mu_{2}+C_{2} \Gamma\left(-Y_{2}\right)\left[\left(G_{2}-\left(\theta_{2}^{*}-1\right)\right)^{Y_{2}}\right.\right.\right. \\
& \quad+\left(G_{2}-\theta_{2}^{*}\right)^{Y_{2}}+\left(2 \theta_{2}^{*}-1\right)\left(G_{2}+1\right)^{Y_{2}}-\left(2 \theta_{2}^{*}+1\right) G_{2}^{Y_{2}}+\left(M_{2}+\theta_{2}^{*}-1\right)^{Y_{2}} \\
& \left.\left.\left.\quad+\left(M_{2}+\theta_{2}\right)^{Y_{2}}+\left(2 \theta_{2}^{*}-1\right)\left(M_{2}-1\right)^{Y_{2}}-\left(2 \theta_{2}^{*}+1\right) M_{2} Y_{2}\right]\right)-a\right\}
\end{aligned}
$$




$$
\begin{aligned}
& +\frac{1}{2} t^{2} a\left\{\mu_{1}-r_{1}+C_{1} \Gamma\left(-Y_{1}\right)\left[\left(G_{1}-\left(\theta_{1}-1\right)\right)^{Y_{1}}-\left(G_{1}+1\right)^{Y_{1}}+G_{1}^{Y_{1}}+M_{1}^{Y_{1}}\right.\right. \\
& \left.\left.-\left(G_{1}-\theta_{1}\right)^{Y_{1}}+\left(M_{1}+\theta_{1}-1\right)^{Y_{1}}-\left(M_{1}-1\right)^{Y_{1}}-\left(M_{1}+\theta_{1}\right)^{Y_{1}}\right]\right\}=0 .
\end{aligned}
$$

Proof In this case, (2.40) and (2.41) are reduced to

$$
\begin{aligned}
\tilde{\xi}_{i}\left(\theta_{i}^{*}(t)\right)-\xi_{i}\left(\theta_{i}^{*}(t)\right)= & \mu_{i}(t)-r_{i}(t)+C_{i} \Gamma\left(-Y_{i}\right)\left[\left(G_{i}-\left(\theta_{i}^{*}(t)-1\right)\right)^{Y_{i}}+G^{Y_{i}}\right. \\
& -\left(G_{i}+1\right)^{Y_{i}}+M^{Y_{i}}-\left(G_{i}-\theta_{i}^{*}(t)\right)^{Y_{i}}+\left(M_{i}+\theta_{i}^{*}(t)-1\right)^{Y_{i}} \\
& \left.-\left(M_{i}-1\right)^{Y_{i}}-\left(M_{i}+\theta_{i}^{*}(t)\right)^{Y_{i}}\right]
\end{aligned}
$$

and

$$
\begin{aligned}
\tilde{\xi}_{i}\left(\theta_{i}^{*}(t)\right)+\xi_{i}\left(\theta_{i}^{*}(t)\right)= & \mu_{i}(t)-r_{i}(t)-2 \theta_{i}^{*}(t) \mu_{i}(t)+C_{i} \Gamma\left(-Y_{i}\right)\left[\left(G_{i}-\theta_{i}^{*}(t)\right)^{Y_{i}}\right. \\
& +\left(G_{i}-\left(\theta_{i}^{*}(t)-1\right)\right)^{Y_{i}}+\left(2 \theta_{i}^{*}(t)-1\right)\left(G_{i}+1\right)^{Y_{i}} \\
& +\left(M_{i}+\theta_{i}^{*}(t)-1\right)^{Y_{i}}+\left(M_{i}+\theta_{i}(t)\right)^{Y_{i}}-\left(2 \theta_{i}^{*}(t)+1\right) G_{i} Y_{i} \\
& \left.+\left(2 \theta_{i}^{*}(t)-1\right)\left(M_{i}-1\right)^{Y_{i}}-\left(2 \theta_{i}^{*}(t)+1\right) M_{i}^{Y_{i}}\right],
\end{aligned}
$$

respectively and the result follows.

The solutions to the martingale condition for $\mathbb{Q}_{\theta}$ are generally not unique and therefore we need to use some criteria to select the final Esscher parameters. These criteria are discussed in the "Appendix".

\section{Numerical Results and Discussions}

In this section, we conduct numerical experiments for the models discussed in the previous sections; the regime switching Black-Scholes (Model I) and CGMY (Model II). We shall assume that there are two states of the economy i.e., $N=2$. State 1 represents an expansion period while state 2 represents a recession period. We assume that the transition probability matrix is

$$
\mathbf{A}=\left(\begin{array}{ll}
-a_{1} & a_{1} \\
a_{2} & -a_{2}
\end{array}\right), \text { with } a_{1}=a_{2}=0.5
$$

\subsection{Model I}

We assume that the stock price is driven by a regime switching geometric Brownian motion. 
Specific Forms of Time Dependent Interest Rate and Volatility. Here, we will extend the results and analysis in Siu and Yang (2009) to the time dependent interest rate and volatility that is, there are both functions of time. We refer the reader to Siu and Yang (2009) (see also Momeya and Morales 2014) in the case of constant parameters.

In the following graphs, it is assumed that the exercise price is 100 , the value of the asset is 120 , and the expiry date is one year in the future. $t=T$ is known as the remaining life of an option. It is also assumed that there is a gradual trend for the parameter to move in a decreasing or increasing manner which might conveniently be regarded as continuous.

We write the two forms as,

(a) Constant model. The interest rates in the two regimes are given by

$$
r_{1}(t)=a_{1} \text { and } r_{2}(t)=a_{2}
$$

(b) Linear model. The interest rates are given by

$$
r_{1}(t)=a_{1}+b_{1} t \text { and } r_{2}(t)=a_{2}-b_{2} t,
$$

where $a_{1}, a_{2}, b_{1}, b_{2}$ are constants with $a_{1}=a_{2}=b_{1}=b_{2}=0.05$.

We define the forms of volatility as;

(a) Constant model. Volatility in the two regimes are given by

$$
\sigma_{1}(t)=b_{1} \text { and } \sigma_{2}(t)=b_{4}
$$

(b) Decaying model. The volatility is given by

$$
\sigma_{1}(t)=b_{1}+b_{2} e^{-b_{3} t} \text { and } \sigma_{2}(t)=b_{4}+b_{5} e^{-b_{6} t} \text {, }
$$

where $b_{1}, b_{2}, b_{3}, b_{4}, b_{5}, b_{6}$ are constants with $b_{1}=0.15, b_{2}=b_{5}=b_{4}=0.25$ and $b_{3}=b_{6}=4$.

In Fig. 1, while keeping the volatility constant, we investigate the impact on the option price of a variation in the form of interest rate when there is no regime (NR), the regime risk not priced (RNP) and the regime risk priced (RP), respectively. In Fig. 2, the same study is made assuming that the interest rate is constant and the form of the volatility can change. Finally, in Fig. 3, we looked at the impact of both linear interest rate and decaying volatility on the option prices in the case of NR, RNP and RP.

As shown in the graphs, the same qualitative results are observed over the lifetime of the option. The initial price of the option is affected in all the situations (NR, RNP and RP) by the change in the form of interest rate and volatility. When the interest is constant, the option price values are very closed during the option's lifetime irrespective of the form of volatility. Note also that, when the regime risk is priced, the option prices are lower when the parameters are time dependent than those with constant parameters. The graphs also show that taking only into account the impact of the regime on the option prices leads to a completely different overall result. For 


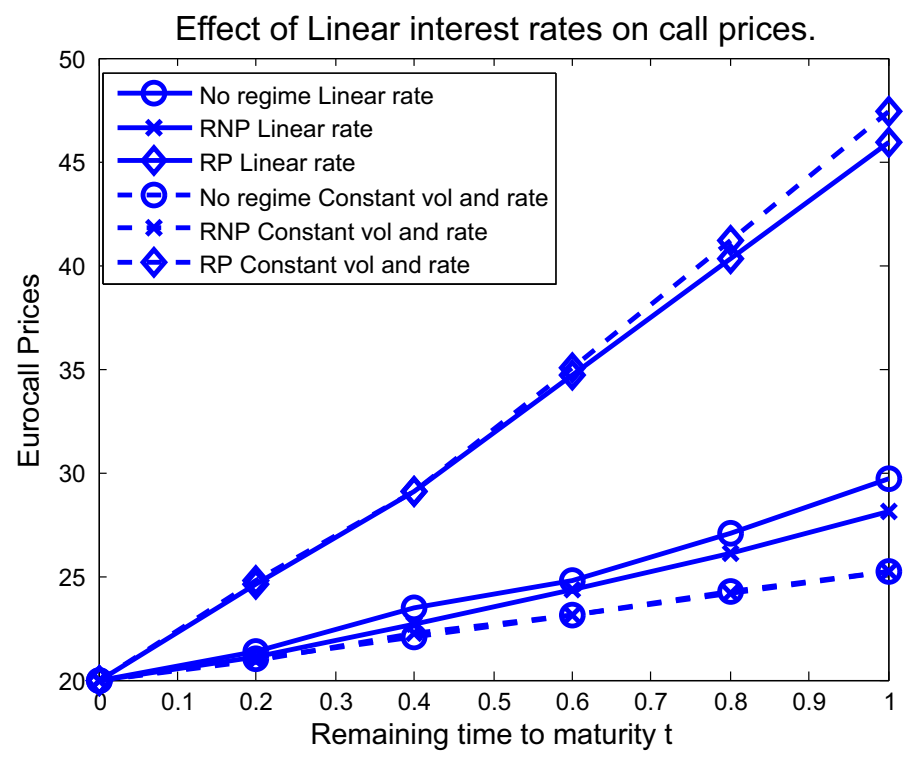

Fig. 1 Effect of linear interest rates

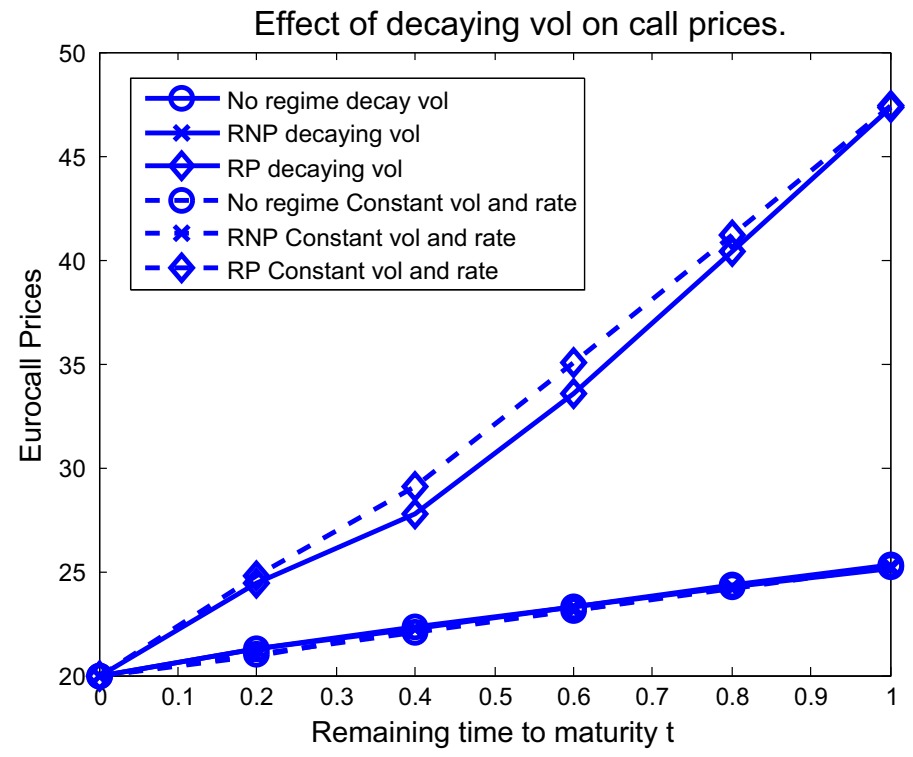

Fig. 2 Effect of decaying volatility

example, the initial value of the option prices are increased substantially when the regime risk is priced. Moreover, during the lifetime of the option, the option prices with the regime risk priced are higher than those with regime risk not priced which are higher than those without regime risk considered. 


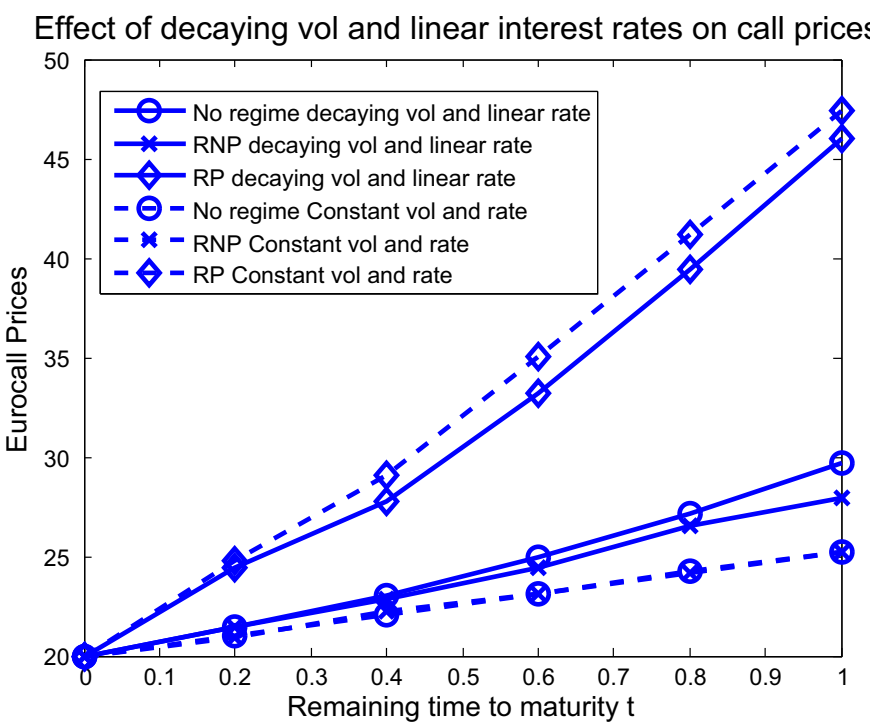

Fig. 3 Effect of linear interest and decaying volatility

\subsection{Model II}

In this section, we discuss the regime switching CGMY model. We cover in particular two cases: $Y=0$ (known as the variance gamma (VG) model) and $Y \neq 0$. We refer the reader to Momeya and Morales (2014) for the case $Y=0$ with constants coefficients.

\subsubsection{VG Case}

We consider linear interest rates and analyse their effects on the call prices. We set

$$
\begin{aligned}
& r_{1}=0.05+0.05 t \text { and } r_{2}=0.01-0.005 t, \\
& C=[3,4], \quad G=[5,6], \quad M=[10,8], \\
& S(0)=100, \quad X(0)=\mathbf{e}_{1}, \quad \mu=[0.35,0.05] .
\end{aligned}
$$

We use the constant parameter case i.e., constant interest rates, as a marker. We define $t=T$ as the remaining time to maturity. We present the results of our simulation below.

In Figs. 4 and 5, we investigate the impact of a variation in the form of interest rate on the option price in three cases: no regime (NR), regime risk not priced (RNP) and regime risk priced (RP). The same conclusions as in the Black-Scholes regime switching model hold concerning the impact of the regime risk on the option prices. Note however that during the life time of the option, the difference in option prices when the regime is priced and when it is not are not significant. 


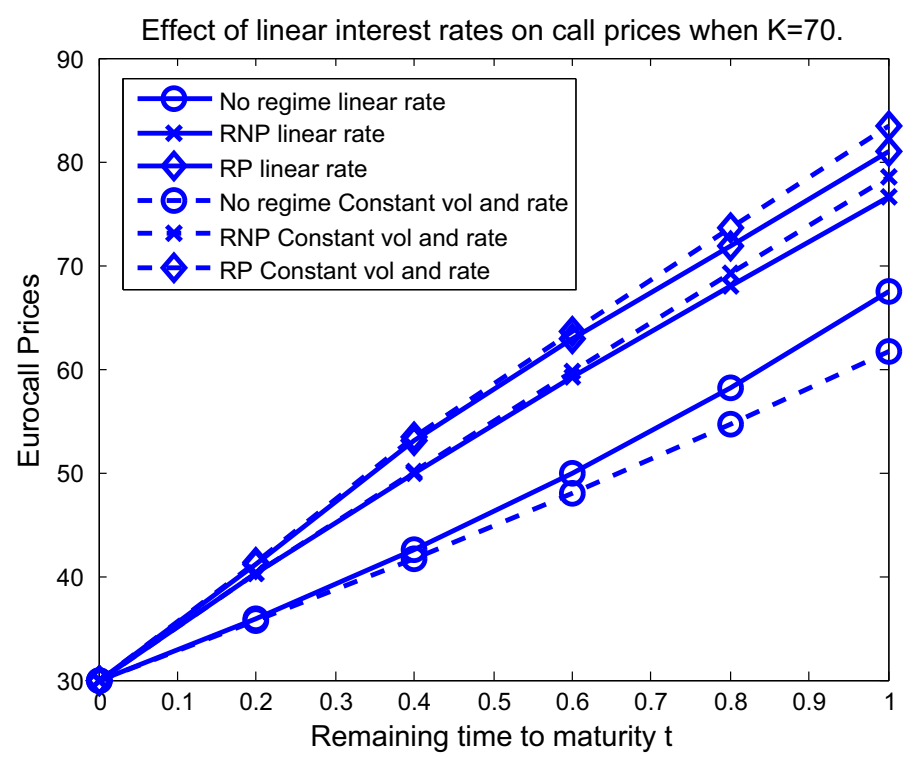

Fig. 4 Effect of Linear rates on call prices when $K=70$

Effect of linear interest rates on call prices when $\mathrm{K}=100$.

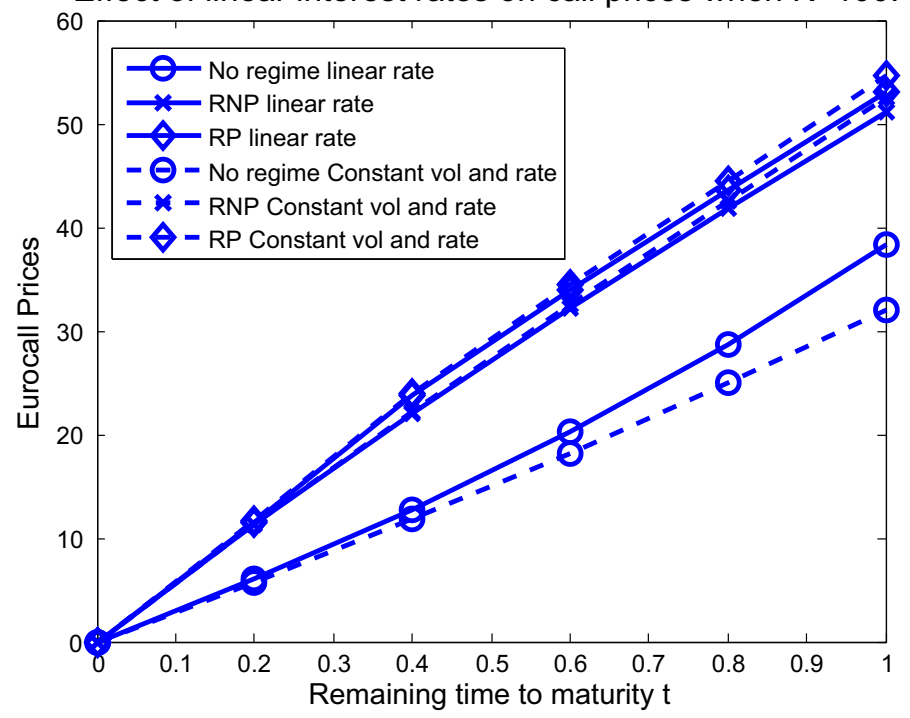

Fig. 5 Effect of Linear rates on call prices when $K=100$

\subsubsection{CGMY Case}

The simulation of this case proved to be more difficult than the former case. We have simulated the CGMY's with $Y>0$. We shall give the results of our simulation in 


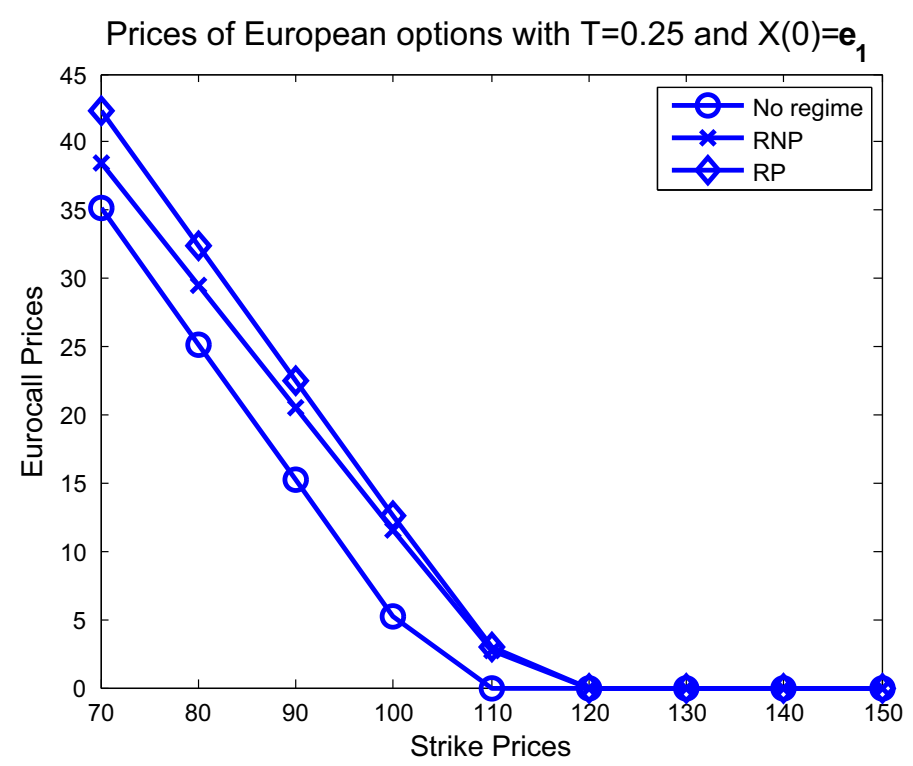

Fig. 6 Call prices across strikes when $T=0.25$

two examples. An algorithm for the simulation of the CGMY process can be found in Cont and Tankov (2004).

(1) We assume that $Y \in(0,1)$ and set the parameters to be

$$
\begin{aligned}
& r=[0.05,0.01], \quad \mu=[0.35,0.05] \\
& C=[3,4], \quad G=[5,6], \quad M=[10,8], \quad Y=[0.5,0.5] \\
& S(0)=100, \quad X(0)=\mathbf{e}_{1}, \quad K=\{70,80,90,100,110,120,130,140,150\}
\end{aligned}
$$

We plot graphs of Call prices across different strikes.

Figures 6, 7, 8 and 9, depict the impact of a change in the regime on the option prices when the strike price changes and the interest rate is constant in three situations: no regime (NR), regime risk not priced (RNP) and regime risk priced (RP). The effect of the parameter $Y$ is seen in this case. As shown in the graph, when the exercised time increases, the initial price of the option is substantially affected. For each time to maturity, as the strike price increases, the value of the option decreases. Contrarily to the Black-Scholes regime switching model (see Siu and Yang 2009), the option prices with regime risk priced are higher than those with regime risk not priced regardless of the option maturity.

Assume now that the interest rates are linear and set

$$
r_{1}=0.05+0.05 t \text { and } r_{2}=0.01-0.005 t
$$

We use the constant parameter case., i.e constant interest rates, as a marker. 


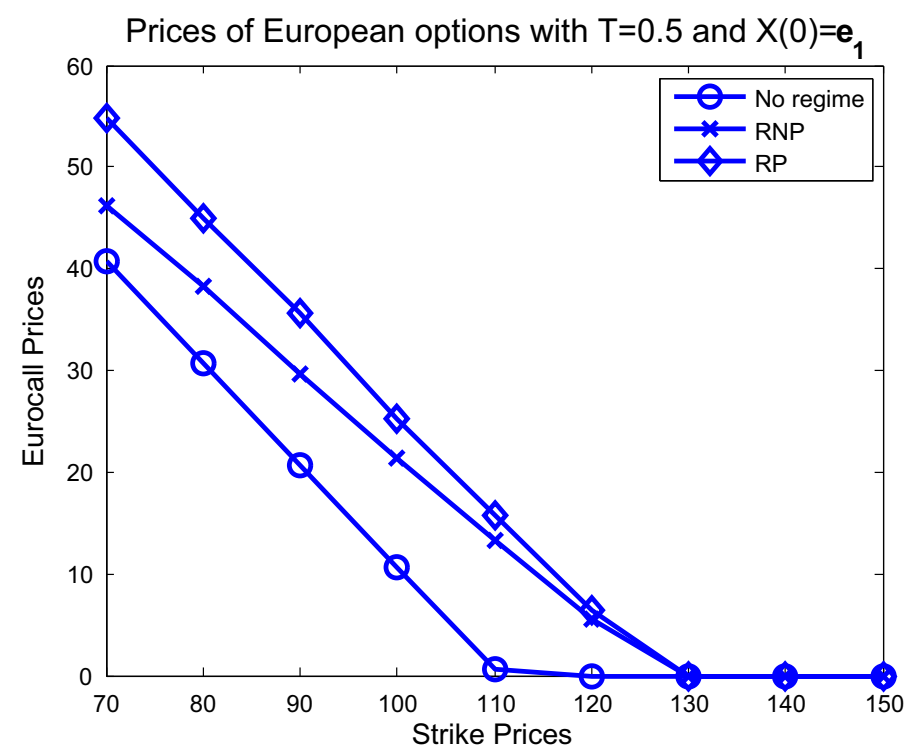

Fig. 7 Call prices across strikes when $T=0.5$

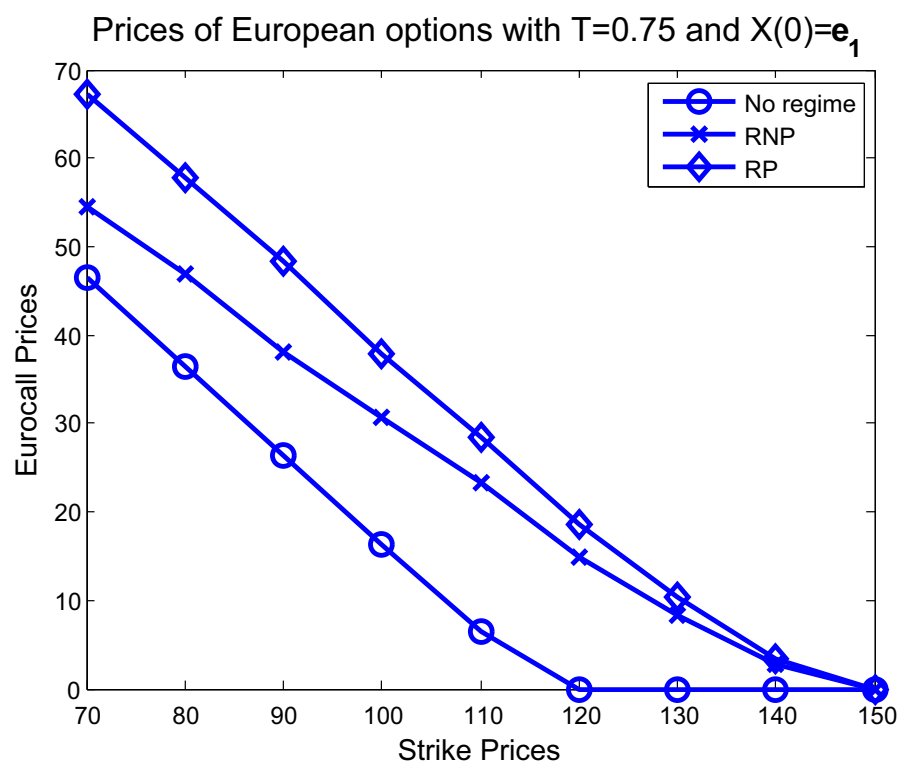

Fig. 8 Call prices across strikes when $T=0.75$

In Figs. 10 and 11, we examine the impact that a change in the form of interest rate has on the option price. It can be seen that, there is no substantial impact of the form of interest rate in the three cases. However, there is a significant difference in the option prices when considering the impact of the regime risk. Once again, the initial value of 


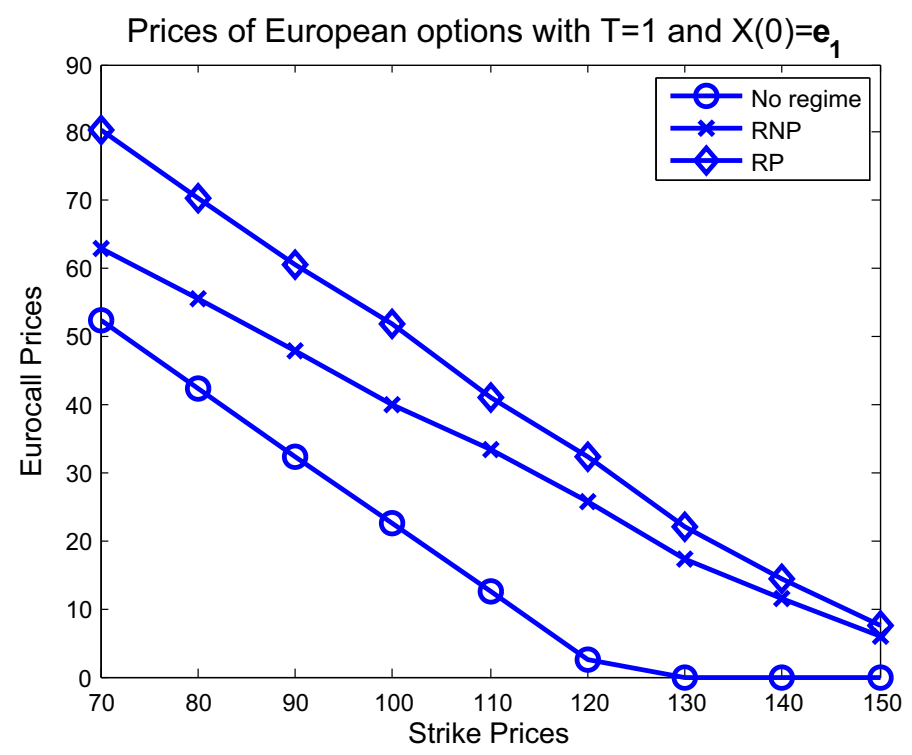

Fig. 9 Call prices across strikes when $T=1$

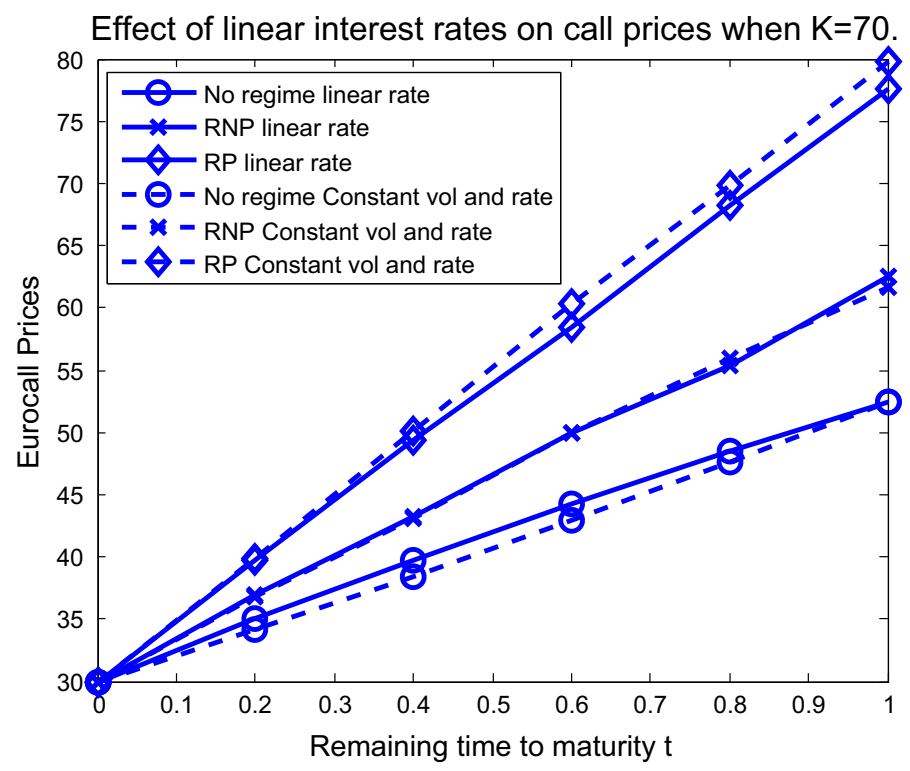

Fig. 10 Effect of Linear rates on call prices when $K=70$

the option price is considerably increased when the regime risk is priced, and during the lifetime of the option, its price when the regime risk is priced is higher than that when the regime risk is not priced which is higher than that when there is no regime. 


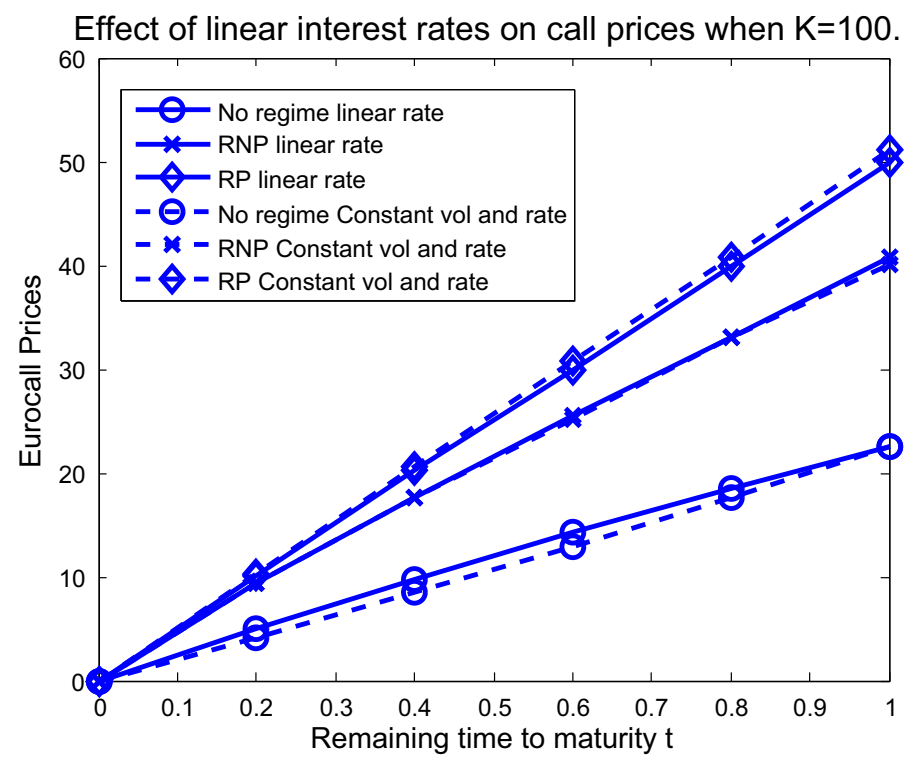

Fig. 11 Effect of Linear rates on call prices when $K=100$

Remark 3.1 When $Y \in(0,1)$, the CGMY process is an infinite activity and finite variation process. This means that the path of the process has a similar behaviour to the path of the VG process.

\section{Conclusion}

In this paper, we use the pricing method developed in Siu and Yang (2009) to price options when the underlying assets are driven by a regime switching CGMY process with time dependent parameters. The theoretical results are given for general regime switching exponential Lévy model with time dependent parameters. The choice of the martingale pricing measure is justified by the minimization of the maximum entropy. We conduct numerical experiments to investigate the effect of pricing regime-switching risk and the analysis shows a significant difference of values between prices of an European call when the regime risk priced and when the regime risk is not priced. We also observe that the regime risk is sensitive to market parameters like time dependent interest rates and volatilities with the sensitivity higher in the case of the Black-Scholes than in the Variance Gamma or CGMY cases.

We may explore the applications of our models to other types of options such as American options, barrier options, look back options, Asian options, Exotic options, option-embedded insurance products, etc. We may also extend our framework to include stochastic interest rates and volatility which would probably give higher values of the option prices. 
Open Access This article is distributed under the terms of the Creative Commons Attribution 4.0 International License (http://creativecommons.org/licenses/by/4.0/), which permits unrestricted use, distribution, and reproduction in any medium, provided you give appropriate credit to the original author(s) and the source, provide a link to the Creative Commons license, and indicate if changes were made.

\section{Appendix 1: Criteria for Selecting Esscher Parameters}

As already mentioned systems of equations characterizing martingale condition for $\mathbb{Q}_{\theta^{*}}$ have in general more than one solution in $\left(\theta_{1}^{*}(t), \theta_{2}^{*}(t)\right)$. Here we present the selection criteria of the set of neutral Esscher parameters $\left(\theta_{1}^{*}(t), \theta_{2}^{*}(t)\right)$ that minimizes the maximum entropy between an EMM and the real world probability measure over different states. The idea is from Siu and Yang (2009).

Define first the entropy between $\mathbb{Q}_{\theta^{*}}$ and $\mathbb{P}$ conditional on $X(0) \in\left\{\mathbf{e}_{1}, \mathbf{e}_{2}\right\}$ as follows:

$$
\begin{aligned}
I\left(\mathbb{Q}_{\theta^{*}}, \mathbb{P}\right):= & E^{\mathbb{P}}\left[\frac{\mathrm{d} \mathbb{Q}_{\theta^{*}}}{\mathrm{~d} \mathbb{P}} \ln \left(\frac{\mathrm{d} \mathbb{Q}_{\theta^{*}}}{\mathrm{~d} \mathbb{P}}\right) \mid X(0)=\mathbf{e}_{i}\right]=E^{\mathbb{P}}\left[\Lambda_{T}^{\theta^{*}} \ln \Lambda_{T}^{\theta^{*}} \mid X(0)=\mathbf{e}_{i}\right] \\
= & \frac{E^{\mathbb{P}}\left[-\int_{0}^{T} \theta(s) \mathrm{d} Y(s) e^{-\int_{0}^{T} \theta(s) \mathrm{d} Y(s)} \mid X(0)=\mathbf{e}_{i}\right]}{E^{\mathbb{P}}\left[e^{-\int_{0}^{T} \theta(s) \mathrm{d} Y(s)} \mid X(0)=\mathbf{e}_{i}\right]} \\
& -\ln E^{\mathbb{P}}\left[e^{-\int_{0}^{T} \theta(s) \mathrm{d} Y(s)} \mid X(0)=\mathbf{e}_{i}\right] .
\end{aligned}
$$

Let $\Gamma:=\left\{\theta^{*} \in \mathbb{R}^{2} \mid \theta^{*}\right.$ satisfies (2.38) and (2.39) $\}$ and denote by $I_{M}\left(\mathbb{Q}_{\theta^{*}}, \mathbb{P}\right)$ the maximum entropy between $\mathbb{Q}_{\theta^{*}}$ and $\mathbb{P}$ over the different values of $X(0)$, i.e.,

$$
I_{M}\left(\mathbb{Q}_{\theta^{*}}, \mathbb{P}\right):=\max _{i=1,2} I\left(\mathbb{Q}_{\theta^{*}}, \mathbb{P} \mid X(0)=\mathbf{e}_{i}\right) .
$$

One can show as in Siu and Yang (2009) that

$$
\begin{aligned}
I\left(\mathbb{Q}_{\theta^{*}}, \mathbb{P} \mid X(0)=\mathbf{e}_{i}\right):= & E^{\mathbb{P}}\left[\frac{\mathrm{d} \mathbb{Q}_{\theta^{*}}}{\mathrm{~d} \mathbb{P}} \ln \left(\frac{\mathrm{d} \mathbb{Q}_{\theta^{*}}}{\mathrm{~d} \mathbb{P}}\right) \mid X(0)=\mathbf{e}_{i}\right] \\
= & \frac{\left\langle e^{\int_{0}^{T}\left(A+\operatorname{diag}\left(\xi_{i}^{k}\left(\theta_{i}^{*}(t)\right)\right)\right) \mathrm{d} t} X(0), \mathbf{1}_{2}\right\rangle}{\left\langle e^{\int_{0}^{T}\left(A+\operatorname{diag}\left(\xi_{i}\left(\theta_{i}^{*}(t)\right)\right)\right) \mathrm{d} t} X(0), \mathbf{1}_{2}\right\rangle} \\
& -\ln \left\langle e^{\int_{0}^{T}\left(A+\operatorname{diag}\left(\xi_{i}\left(\theta_{i}^{*}(t)\right)\right)\right) \mathrm{d} t} X(0), \mathbf{1}_{2}\right\rangle .
\end{aligned}
$$

The selected $\left(\theta_{1}^{*}(t), \theta_{2}^{*}(t)\right)$ shall be solution to the following problem: Find $\left(\hat{\theta}_{1}^{*}(t), \hat{\theta}_{2}^{*}(t)\right) \in \Gamma$ such that

$$
I_{M}\left(\mathbb{Q}_{\hat{\theta}^{*}}, \mathbb{P}\right)=\min _{\theta^{*} \in \Gamma} I_{M}\left(\mathbb{Q}_{\theta^{*}}, \mathbb{P}\right)
$$

with $\Gamma:=\left\{\theta^{*} \in \mathbb{R}^{2} \mid \theta^{*}\right.$ satisfies (2.38) and (2.39) $\}$. 


\section{Appendix 2: Simulation Procedure}

In this section, we discuss the simulation procedure. We adopt a straight forward Monte-Carlo procedure in order to obtain simulation approximations for the European call price. Suppose we want to evaluate the price of a European call option at the current time $t=0$ with maturity $T$ and strike price $K$. We note that the call option $C(0, S(0), X(0))$ can be evaluated as follows:

$$
\begin{aligned}
C(0, X(0), S(0)) & =E^{\theta^{*}}\left[\exp \left(-\int_{0}^{t} r(u) \mathrm{d} u\right)(S(T)-K)^{+}\right] \\
& =E^{\mathbb{P}}\left[\frac{\mathrm{d} \mathbb{Q}_{\theta^{*}}}{\mathrm{~d} \mathbb{P}} \exp \left(-\int_{0}^{t} r(u) \mathrm{d} u\right)(S(T)-K)^{+} \mid S(0), X(0)\right] .
\end{aligned}
$$

We assume that the process $S$ is simulated over a discrete grid. To achieve this, we divide the time horizon $[0, T]$ into $J$ subintervals $\left[t_{j}, t_{j+1}\right]$ for $j=0,1, \ldots, J-1$ of equal length $\Delta=\frac{T}{J}$ where $t_{0}=0$ and $t_{J}=T$.

For the discrete-time version of the Markov chain $X$, we suppose that the transition probability matrix in a subinterval is $I+\mathbf{A} \Delta$ given $X(0)$.

Given the simulated path of $X$, the sample paths of the processes $\left\{\mu\left(t_{j}\right)\right\}_{j=1}^{J}$, $\left\{\sigma\left(t_{j}\right)\right\}_{j=1}^{J},\left\{\theta\left(t_{j}\right)\right\}_{j=1}^{J}$ and $\left\{r\left(t_{j}\right)\right\}_{j=1}^{J}$ are identified. Then, we can now use these to construct a Euler forward discretization scheme to discritize the log return process $Y$ as follows

$$
\begin{aligned}
Y\left(t_{j+1}\right)= & Y\left(t_{j}\right)+\Delta *\left(\mu\left(t_{j}\right)-\frac{1}{2} \sigma^{2}\left(t_{j}\right)\right)+\Delta * \int_{\mathbb{R}}\left(e^{z}-1-z\right) \rho^{X\left(t_{j}\right)}(d z) \\
& +\sigma\left(t_{j}\right) * \xi * \sqrt{\Delta}+\tilde{J}_{j}^{X}\left(t_{j+1}\right)-\tilde{J}_{j}^{X}\left(t_{j}\right) .
\end{aligned}
$$

where $\xi \sim N(0,1)$ and

$$
\tilde{J}_{j}^{X}(t)=\int_{\mathbb{R}} z J_{j}^{X}(t ; \mathrm{d} z)-\int_{0}^{t} \int_{\mathbb{R}} z \rho^{X\left(t_{j}\right)}(\mathrm{d} z) \mathrm{d} t .
$$

Given $\left\{X\left(t_{j}\right)\right\}_{j=1}^{J}$ and $Y(0)=0$, we then sample $\left\{Y\left(t_{j}\right)\right\}_{j=1}^{J}$ using (4.6) recursively. The Monte Carlo simulation procedure can be found in Siu and Yang (2009).

\section{References}

Arai, T. (2001). The relations between minimal martingale measure and minimal entropy martingale measurenimal martingale measure and minimal entropy martingale measure. Asia-Pacific Financial Markets, 8(2), 167-177.

Black, F., \& Scholes, M. (1973). The pricing of options and corporate liabilities. The Journal of Political Economy, 75, 637-659.

Cont, R., \& Tankov, P. (2004). Financial modeling with jump processes. UK: Chapman \& Hall/CRC Financial Mathematics Series. 
Elliott, R. J., Aggoun, L., \& Moore, J. B. (1994). Hidden Markov models: Estimation and control. New York: Springer.

Elliott, R. J., Chan, L., \& Siu, T. K. (2005). Option pricing and Esscher transform under regime switching. Annals of Finance, 1, 423-432.

Elliott, R. J., \& Osakwe, C. J. (2006). Option pricing for pure jump processes with Markov switching compensators. Finance and Stochastics, 10, 250-275.

Elliott, R. J., \& Siu, T. K. (2011). Pricing and hedging contingent claims with regime switching risk. Communications in Mathematical Sciences, 9, 477-498.

Elliott, R. J., Siu, T. K., \& Badescu, A. (2011). On pricing and hedging options under double Markovmodulated models with feedback effect. Journal of Economics Dynamics and Control, 35, 694-713.

Fujisak, M., \& Zhang, D. (2009). Evaluation of the MEMM, parameter estimation and option pricing for geometric Lévy processes. Asia-Pacific Financial Markets, 16(2), 111-139.

Fujiwara, T. (2006). From the minimal entropy martingale measures to the optimal strategies for the exponential utility maximization: The case of geometric Lévy processes. Asia-Pacific Financial Markets, 11(4), 367-391.

Gerber, H. U., \& Shiu, E. S. (1994). Option pricing by Esscher transforms (with discussions). Transactions of the Society of Actuaries, 46, 99-191.

Goldfeld, S., \& Quandt, R. E. (1973). A Markov model for switching regressions. Journal of econometrics, 1, 3-15.

Hamilton, J. D. (1989). A new approach to the economics analysis of non-stationary time series. Econometrica, 57, 357-384.

Harrison, J., \& Pliska, S. (1981). Martingales and stochastic integrals in the theory of continuous trading. Stochastic Processes and their Applications, 11, 215-260.

Harrison, J., \& Pliska, S. (1983). A stochastic calculus model of continuous trading: Complete markets. Stochastic Processes and their Applications, 15, 313-316.

Hyland, K., Mckee, S., \& Waddell, C. (1999). Option pricing, Black-Scholes, and novel arbitrage possibilities. IMA Journal of Mathematics Applied in Business \& Industry, 10, 177-186.

Merton, R. C. (1973). The theory of rational option pricing. Bell Journal of Economics and Management Science, 4, 141-183.

Miyahara, Y. (1999). Minimal entropy martingale measures of jump type price processes in incomplete assets markets. Asia-Pacific Financial Markets, 6(2), 97-113.

Momeya, R., \& Ben-Salah, Z. (2012). The minimal entropy martingale measure (MEMM) for a MarkovModulated exponential Lévy model. Asia-Pacific Financial Markets, 19(1), 63-98.

Momeya, R., \& Morales, M. (2014). On the price of risk of the underlying Markov chain in a regimeswitching exponential Lévy model. Methodology and Computing in Applied Probability. doi:10.1007/ s11009-014-9399-2.

Naik, V. (1993). Option valuation and hedging strategies with jumps in the volatility of asset returns. Journal of Finance, 48, 1969-1984.

Siu, T. K., \& Yang, H. (2009). Option pricing when the regime-switching risk is priced. Acta Mathematicae Applicatae Sinica, 3, 369-388. 